\title{
ESTÁNDARES DE EGRESO Y CERTIFICACIÓN INICIAL DOCENTE: LA EXPERIENCIA INTERNACIONAL ${ }^{1}$
}

\section{Lawrence Ingvarson ${ }^{2}$}

\section{RESUMEN}

Este artículo presenta las características principales de la experiencia internacional en la formulación de estándares de egreso y certificación inicial docente, revisando entre otros los casos de Australia, Arabia Saudita, Inglaterra, Nueva Zelanda, Escocia, Estados Unidos y Chile. El artículo expone la relevancia de promover la calidad docente; explica los procesos de descripción y fijación de estándares; el desarrollo de los estándares de contenido; los estándares genéricos y los estándares específicos según el contenido y el nivel de enseñanza. Finalmente da cuenta del proceso de formulación de estándares para docentes en Chile.

Palabras clave: Estándares, calidad, formación docente

\section{STANDARDS ON GRADUATION AND INITIAL TEACHER CERTIFICATION: THE INTERNATIONAL EXPERIENCE}

\begin{abstract}
This article describes the main characteristics of international experience in formulating standards on graduation and initial teacher certification. It also reviews, among others, cases of Australia, Saudi Arabia, England, New Zealand, Scotland, United States and Chile. It emphasizes the importance of promoting quality in teaching and explains the processes of describing and fixing standards, the development of standards on content, and generic standards and specific standards according to content and teaching level. It ends with a description of the process of formulating standards for teaching here in Chile.
\end{abstract}

Keywords: Standards, quality, teacher preparation

1 Documento elaborado para la participación en el XVII Seminario internacional CNED «Nuevos horizontes en la formación docente: El desafío de contar con los profesores que Chile necesita», realizado el 31 de octubre de 2012.

2 Australian Council for Educational Research, Melbourne, Australia. Contacto: lawrence.ingvarson@acer.edu.au 


\section{ESTÁNDARES DE EGRESO Y CERTIFICACIÓN INICIAL DOCENTE: LA EXPERIENCIA INTERNACIONAL}

\section{Promover la calidad docente}

En los últimos años ha crecido notablemente el interés internacional en políticas que promuevan la calidad docente (OCDE, 2005). Con pruebas crecientes de que lo más importante en la influencia de la escuela por sobre el logro estudiantil es el conocimiento y las habilidades de los profesores (Hattie, 2008; Hanushek, 2004), las autoridades responsables de la formulación de políticas están poniendo más atención en estrategias que recluten, preparen y retengan a los mejores profesores posibles. Estas estrategias requieren descripciones claras y válidas acerca de lo que saben y hacen los buenos profesores, mediante la preparación de estándares para la docencia.

Cuando se trata de la calidad de las oportunidades para que los estudiantes aprendan en las escuelas, la investigación es clara: nada es tan fundamental como el conocimiento profesional, el criterio y las habilidades de sus profesores. Varios estudios, como el de McKinsey y Company (Barber y Mourshed, 2007), muestran que los sistemas escolares con mejor desempeño en el mundo, como los de Finlandia, Singapur y Corea del Sur, dan prioridad a las políticas, estrategias e instituciones para contratar, preparar y reconocer a profesores de calidad.

La profesión docente y las escuelas de un país constituyen una infraestructura tan importante en la viabilidad económica de largo plazo, como los tipos de infraestructura más obvios como por ejemplo la producción de energía y el transporte. Por ello, esta se debe nutrir con afirmaciones y reabastecer en el largo plazo. No existen los atajos para construir un sistema escolar de alta calidad: países como Finlandia, Corea del Sur, Singapur y Taiwán, que suelen tener excelentes evaluaciones en pruebas internacionales de logro académico, han perseguido siempre políticas que mejoren la calidad 
de sus profesores desde la Segunda Guerra Mundial; otros en cambio han abandonado lo que era un activo importante y han dejado caer el estatus y la calidad de los profesores.

En el ámbito internacional, los objetivos curriculares para estudiantes dejan en claro que el conocimiento disciplinario que se exige a los profesores va en aumento. Los currículos nacionales son cada vez más ambiciosos en relación con lo que los docentes deben lograr. El modelo pedagógico antiguo, que aseguraba que la enseñanza era algo que casi cualquiera podía hacer bien, ya no funciona. Nuevos informes de la OCDE (OCDE, 2010; 2011) señalan que la mejor forma de competir en la economía global es ofrecer a todos los ciudadanos la calidad de educación que antes se destinaba solo a la élite. Esto no sucederá a menos que un país tenga una sólida disposición a elevar la calidad de sus profesores.

Las políticas que promueven la calidad docente deben operar en varias etapas, desde la contratación misma hasta la retención de buenos profesores. Un nuevo estudio del IEA, Teacher education and development study in mathematics (TEDS-M) (Tatto, Schwille, Senk, Ingvarson, Rowley, Peck, Bankov, Rodríguez y Reckase, 2012), muestra la importancia de la coordinación entre las políticas diseñadas para garantizar la calidad docente. El estudio TEDS-M recopiló datos respecto de las disposiciones de aseguramiento de la calidad en diecisiete países en las cuatro siguientes etapas:

a) Contratación y selección: El objetivo está puesto en las políticas y organismos que tiene un país para monitorear y garantizar la calidad de quienes desean formarse como profesores. En particular, políticas en relación con:

- matrícula en formación docente;

- hacer que la docencia sea una opción de carrera atractiva y

- exigencias de contenido para la admisión en la formación docente.

b) Acreditación de instituciones de formación docente: El objetivo está puesto en políticas y organismos que monitoreen y garanticen la calidad de las instituciones de formación docente y sus programas. 
c) Ingreso a la profesión docente: El objetivo está puesto en políticas y organismos que garanticen que los egresados sean competentes y estén calificados antes de obtener su certificación y el ingreso pleno a la profesión.

d) Certificación avanzada: El objetivo está puesto en políticas y sistemas que definan los estándares para la docencia de alta calidad y ofrezcan incentivos para que la mayoría de los profesores alcancen dichos estándares.

Estos son los cuatro mecanismos principales mediante los cuales los países buscan garantizar la calidad de los futuros profesores. Juntos forman un conjunto coherente de políticas para promover la calidad docente.

El estudio IEA TEDS-M encontró una relación importante entre el rigor de las disposiciones para el aseguramiento de la calidad y la calidad de los egresados de programas de formación docente, según miden las pruebas de conocimiento matemático y de conocimiento del contenido matemático utilizadas en TEDS-M (Ingvarson, Schwille, Rowley, Tatto, Peck, Senk, no publicado). Los países con fuertes disposiciones para el aseguramiento de la calidad, como China (Taipéi) y Singapur, tuvieron las mejores evaluaciones en estas mediciones, mientras que los países con disposiciones más débiles, como Georgia y Chile, tendían hacia evaluaciones más bajas en mediciones de conocimiento matemático y de conocimiento del contenido matemático.

El estudio TEDS-M muestra, por ejemplo, que países como China (Taipéi) y Singapur, que tienen buenas evaluaciones en pruebas internacionales de logro académico (por ejemplo TIMSS) no solo garantizan la calidad de quienes ingresan a la formación docente, sino que a la vez tienen buenos sistemas para revisar, evaluar y acreditar a los proveedores de formación docente. También cuentan con buenos mecanismos para asegurar que los egresados cumplan con altos estándares de desempeño antes de obtener certificación y el pleno ingreso a la profesión y, por último, ofrecen sueldos, condiciones laborales y trayectorias relativamente atractivas que recompensan la evidencia de lograr altos estándares para la docencia. 


\subsection{Acreditación e ingreso a la profesión docente}

El interés de este documento se encuentra en la segunda y la tercera etapa, es decir, en las políticas y prácticas para asegurar la calidad de los programas de formación docente y de los profesores que egresan de esos programas antes de obtener el pleno ingreso a la profesión. Los estándares para los profesores nuevos, especificados en el caso de Chile en la publicación Estándares orientadores para egresados de carreras de Pedagogía en Educación Básica, tienen obviamente una función importante que cumplir. El ingreso a la profesión es sin duda uno de los puntos decisivos más importantes para asegurar la calidad docente. Una vez que un profesor ingresa oficialmente al rubro, puede enseñar durante treinta años o más, lo que afectará el desarrollo de cientos de estudiantes. En algunos sistemas escolares podría ser difícil sacar a los profesores incompetentes sin prolongadas acciones legales.

Así, existen varios términos como certificación, registro o aprobación, que se usan para referirse al consenso de que una persona ha alcanzado los estándares para ingresar de lleno a una profesión. Según sea el país, el responsable de este consenso puede ser una agencia de gobierno, una autoridad reglamentaria o una agrupación profesional independiente. El cuerpo de certificación suele ser la misma agencia que es responsable de la acreditación de programas de preparación profesional (por ejemplo, el Consejo General Docente de Escocia).

En el pasado se consideraba que obtener una calificación como docente en una universidad era una base suficiente para obtener la certificación de una agencia de estándares profesionales y la idoneidad para emplearse como profesor en una escuela. Se suponía, igualmente, que el egreso de un programa de formación docente era, por sí mismo, garantía de que un profesor era competente para la docencia, pero esta práctica está disminuyendo. En efecto, en forma cada vez más extensa, los países introducen métodos para determinar si los egresados de los programas de formación docente deberían obtener certificación y pleno ingreso a la profesión.

Así, con el fin de asegurar la calidad de los nuevos profesores, muchos países como Inglaterra, Omán, Filipinas, España y la mayoría 
de los estados de EE.UU. exigen que los egresados den un examen externo (por ejemplo, pruebas de habilidades alfabéticas y numéricas y conocimiento profesional) y/o algún tipo de examen competitivo, además de la obtención de una calificación universitaria. El cuerpo responsable suele ser un organismo de gobierno nacional o estatal.

Una tendencia generalizada en países con una fuerte orientación hacia el aseguramiento de la calidad es exigir a los profesores egresados que aprueben una inducción o periodo de prueba en las escuelas, antes de obtener la certificación y el ingreso pleno a la profesión. Esto ofrece oportunidades para apoyar su desarrollo, evaluar su desempeño y garantizar de varias formas que estos docentes hayan logrado los estándares asignados para el ejercicio.

Australia, Taiwán, Alemania y algunos estados de EE.UU. son ejemplos de países en donde la certificación y/o el ingreso a la profesión no solo depende de aprobar pruebas de conocimiento profesional, sino que además se requieren evaluaciones formales de desempeño durante un periodo de prueba. Estos países tienen organismos separados de las universidades que exigen evaluaciones formales del desempeño en el aula, simultáneamente con los exámenes normales, antes que los nuevos profesores se certifiquen y tengan pleno ingreso a la profesión (como en Australia y EE.UU.), o bien, un cargo en una escuela o el acceso al servicio público (como en Alemania).

Los egresados de los programas de formación docente en Taiwán, por ejemplo, enfrentan un riguroso conjunto de procedimientos de aseguramiento de la calidad antes de obtener finalmente un cargo docente. Después de culminar su programa de formación docente con una nota adecuada para aprobar, los egresados deben dar una Evaluación de Calificación Docente (TQA, por sus siglas en inglés) del Ministerio de Educación. La TQA es una prueba nacional común que se realiza una vez al año, dos meses después de que los estudiantes han terminado la práctica docente, hacia finales de marzo y que es supervisada por el comité de verificación de calificaciones docentes del Ministerio de Educación y una institución administrativa designada por el mismo ministerio diseñan la TQA. 
Si los egresados aprueban la TQA, el ministerio les emite una credencial de profesores y quedan oficialmente calificados para ejercer la docencia. Sin embargo, si postulan a un cargo docente en una región en particular, los profesores calificados deben pasar por un proceso de «filtro» en dos etapas, administrado por el distrito escolar. La primera instancia consiste en pruebas escritas para evaluar el conocimiento profesional docente del postulante y el conocimiento de materias. Solo pocos postulantes pasan la primera ronda. Los egresados que llegan a la segunda ronda son evaluados a través de una demostración de su enseñanza y de una entrevista personal, la que suele ser evaluada por dos grupos de tres a cinco jueces respectivamente (principalmente profesores de la escuela y un director; en ocasiones, docentes universitarios).

Estos sistemas emergentes para asegurar el rigor del proceso inicial de certificación dependen, obviamente, del desarrollo de estándares válidos que describan lo que deberían saber y hacer los profesores principiantes.

A continuación analizaremos el significado de los estándares para la docencia y revisaremos los enfoques internacionales hacia su desarrollo.

\section{El significado de los estándares para la docencia}

Las connotaciones de la palabra estándar son ricas, variadas y rememorativas, con indicios de seguridad, permanencia y calidad. Es probable que esto ayude a considerar su eterna popularidad entre los comentaristas del tema de la educación. Para algunos, los estándares se remontan a tiempos de confianza y certeza, los que han ido decayendo desde los tiempos clásicos, aunque por definición, siempre son levemente atemporales. Para otros, los estándares intentan inspirar describiendo una visión de lo que podría ser. Los estándares orientados hacia el futuro se basan en capturar la esencia de lo mejor que aparece en la práctica y la investigación actuales respecto de las buenas prácticas docentes. 
Los estándares se refieren a normas, exigencias, o bien, a la magnitud oficial de una unidad de medición o indicadores de niveles de excelencia profesional. Este documento usará el último significado.

Como puntos de unidad, los estándares buscan articular valores docentes centrales que los profesores deberían manifestar en su práctica. Los creadores de estándares profesionales serán guiados por conceptos de aprendizaje y desarrollo lo que significa, por ejemplo, educar una mente, aprender con comprensión y pensar en forma independiente al profesor. Por definición, los estándares son afirmaciones acerca de lo que se valora.

Como medición, los estándares no solo describirán lo que necesitan saber y hacer los profesores para poner en práctica estos valores, sino que describirán también la forma en la que se evaluará el logro de este conocimiento y lo que cuenta para cumplir con él. Un estándar, en este último sentido, es el nivel de desempeño respecto de los criterios que se evalúan y que se consideran satisfactorios en términos del objetivo de la evaluación.

Ahora bien, es necesario distinguir entre estándares absolutos y estándares relativos. Un estándar absoluto es una evaluación o nivel designado de desempeño que se fija sin referencia a la distribución de individuos en la población que se intenta evaluar y donde se usará el estándar. Por ejemplo, fijar una exigencia de evaluación de un ensayo en 10 puntos dentro de una escala de 12, o bien, exigir un salto con garrocha de cinco metros: en ambos casos estamos hablando de estándares absolutos.

Un estándar relativo, por su parte, es una evaluación de selección o nivel designado de desempeño que se fija, con referencia, a la forma en que otras personas se desempeñan en una evaluación $y$, sin referencia, a un nivel de desempeño absoluto. Por ejemplo, obtener la evaluación en el cuartil más alto en una prueba escrita o estar entre los diez primeros lanzadores del disco en un grupo de 100 personas son estándares relativos, no absolutos.

En un principio, los estándares para la docencia para el registro o certificación avanzada deberían ser estándares absolutos. 
Por ejemplo, evaluar a un profesor en la norma: «El profesor puede organizar un discurso con los estudiantes acerca de ideas científicas» requeriría la creación de una rúbrica de evaluación, es decir, de un conjunto de descriptores, reglas, pautas o referencias para evaluar niveles de desempeño distintos.

En la práctica, los estándares para la docencia, donde los hay, son mayormente estándares relativos, determinados por los factores de oferta y de demanda de profesores o por el número limitado de cargos promovidos disponibles.

\subsection{Describir estándares versus fijar estándares}

Se debe distinguir entre la labor de describir estándares y la de fijar estándares. Describir estándares es un intento de articular el conocimiento profesional y las habilidades que se valoran. Estos se suelen denominar estándares de contenido. Este proceso a menudo depende de reunir a profesionales expertos y, a través de la amplia reiteración de conversaciones, borradores, circulación de comentarios, nuevos borradores, etc., trabajar hacia un consenso acerca de lo que un profesor experto en esa área temática debe saber y debe poder hacer para promover el aprendizaje de calidad.

Fijar estándares, por su parte, representa un ejercicio completamente distinto. La fijación de estándares es un proceso de acordar qué habilidades y conocimientos se deben demostrar para alcanzar un nivel que se considere adecuado: es decir, fijar los estándares de desempeño. Se trata de determinar qué es considerado aceptable para un fin específico y fijar los criterios de rechazo o de aprobación; por ejemplo, la evaluación para decidir si un profesor a prueba ha alcanzado los estándares de registro para el ejercicio docente o si un profesor experimentado ha alcanzado los estándares avanzados establecidos por un cuerpo profesional. Un estándar indica y describe un nivel deseable de desempeño.

En resumen, hay tres pasos prácticos en la creación de los estándares para la evaluación de un profesor: 
- Describir la buena docencia y definir lo que se va a evaluar, es decir, qué saben y hacen los buenos profesores (estos suelen llamarse estándares de contenido).

- Desarrollar métodos de evaluación válidos y coherentes para recopilar evidencia acerca de lo que sabe un profesor y de lo que puede hacer en relación con los estándares.

- Desarrollar procedimientos confiables para evaluar esa evidencia y decidir si un profesor ha cumplido con el estándar (es decir, fijación de estándares de desempeño).

Los estándares para la docencia deben identificar las labores centrales de la enseñanza y adaptarse de vez en cuando al cambio en las expectativas públicas de las escuelas. Algunos dirán que las materias son el núcleo del trabajo de los profesores y que la tarea central de la docencia es ayudar a los diversos estudiantes a aprender materias importantes y, en ese caso, las funciones que cumplen como cuidadores, administradores de aula y modelos de conducta son medios para ese fin.

Los estándares también necesitan identificar las características únicas de lo que saben y hacen los profesores que enseñan disciplinas particulares o que enseñan en niveles determinados de escolaridad. Tradicionalmente, la investigación acerca de la docencia ha buscado principios de buenas prácticas que se apliquen sin importar la materia que se enseñe (Brophy, 1991) y, por otro lado, en los últimos años dicha investigación ha enfatizado la naturaleza altamente específica del contexto del conocimiento disciplinario en docencia, por ejemplo, Shulman (1987).

Los estándares no solo describen la práctica actual, sino que aclaran lo que los profesores deberían saber y hacer a la luz de la investigación y de las mejores prácticas (Dwyer, 1994). De este modo, los estándares representan un puente entre la investigación y la práctica en tanto representan una forma de traducir la investigación en expectativas para la práctica docente. Por otro lado, no son inmutables, lo cual implica que necesitan de una revisión regular a la luz de la investigación y del conocimiento profesional. 
Los estándares para los docentes en ejercicio aclaran aquello en lo que estos deberían mejorar en el largo plazo. Este es el objetivo de los desarrollados por el Directorio Nacional de Estándares Profesionales en EE.UU. Además, los estándares especifican trayectorias para el desarrollo profesional y de las carreras, como lo ilustra el caso de Australia que se abordará después. Ellos ponen de manifiesto la idea de que la buena docencia es algo que una persona aprende a hacer en el tiempo, que no es solo un puñado de rasgos de personalidad, es decir, los estándares rechazan la mentalidad de que la docencia es solo un asunto de estilo personal y de «hacer lo propio».

Finalmente, los estándares dan una garantía a la afirmación de que la docencia es una profesión con la capacidad de evaluar su propia práctica e implementar modelos profesionales de responsabilidad, ofreciendo una base para que los profesores y sus asociaciones muestren liderazgo en su profesión, tal como lo ilustran aquellos desarrollados por las asociaciones de profesores entre las que destacan la Asociación Australiana de Profesores de Ciencias (2002) y el Consejo Nacional de Profesores de Matemáticas en EE.UU. (1989).

\section{Definir la buena docencia: desarrollar estándares de contenido}

Existe una larga historia de esfuerzos para definir qué es una buena docencia. Mary Kennedy (2010) ofrece una revisión acabada de cómo el concepto del «buen profesor» cambió en el último siglo, rastreando el movimiento a favor de los estándares hasta el final del siglo XX como una manera de definir lo que saben y hacen los buenos profesores. Los primeros esfuerzos datan de los años sesenta y se enfocaron en algunos rasgos de la personalidad del profesor, más que en sus prácticas en el aula. A continuación siguió un periodo que duró hasta los años noventa y que estuvo marcado por la investigación procesoproducto, la que se concentraba en identificar los comportamientos específicos que caracterizaban a la docencia eficaz. Ninguno de los dos enfoques resultó satisfactorio en términos de capturar la importancia educacional de lo que estaba sucediendo en las aulas. Los estándares para la docencia demostraron ser una base más sólida sobre la cual juzgar la calidad de las oportunidades que los profesores daban a sus 
estudiantes para que aprendieran, en particular, cuando llamaban la atención hacia la sustancia intelectual de lo que se estaba enseñando, cómo se representaba y cómo los estudiantes interactuaron con eso.

Así, como primer paso en el desarrollo de estándares para la docencia, el equipo a cargo necesitará articular una visión del aprendizaje de calidad que describa en detalle lo que los profesores deberían saber, creer y poder hacer, dado que el objetivo final de los estándares es mejorar la calidad de las oportunidades de aprendizaje para los estudiantes en escuelas. Como señala Kennedy (2010), estos necesitan ser accionados por una visión del aprendizaje de alta calidad acerca de algo, sea ello alfabetismo, números o ciencia, y por la importancia de que los estudiantes aprendan ese algo. En las declaraciones respecto del currículo nacional suele encontrarse una visión contemporánea de lo que necesitan saber y poder hacer los estudiantes.

Un enfoque productivo para iniciar el proceso de escribir estándares es reunir grupos de profesores e investigadores expertos para que analicen, en primer lugar, no la docencia, sino sus visiones acerca de lo que consideran un aprendizaje de calidad en su área de docencia (por ejemplo, docencia primaria, docencia especializada en matemáticas, etc.). Esta conversación será más fluida con preguntas orientadoras como las siguientes:

- ¿Qué considera usted como un aprendizaje de calidad en su área de disciplina?

- ¿Qué veríamos en un aula en donde se lleva a cabo un aprendizaje de calidad?

Las pautas del currículo nacional resultarán útiles para este tipo de conversación. Por ejemplo, el currículo nacional para ciencias en Australia se organiza alrededor de tres tendencias interrelacionadas: la comprensión científica, las habilidades de investigación científica y la ciencia como un esfuerzo humano. Cada una de estas pautas se elabora a su vez bajo la visión de la importancia de aprender ciencia en las escuelas. En la mayoría de los países, el currículo nacional suele ofrecer visiones igualmente inspiradoras para el aprendizaje en varias materias. 
Una vez que el análisis comienza a coincidir respecto de algunas visiones comunes sobre el aprendizaje de calidad, los grupos que escriben los estándares pueden volver a la pregunta central: ¿Qué necesitarían saber y hacer los profesores para promover lo que consideramos como un aprendizaje de calidad en nuestra área de docencia?

Para abordar esta pregunta, los redactores de estándares pueden usar una variedad de fuentes entre las que se cuenta la experiencia y la sabiduría de profesores expertos y la investigación respecto de prácticas docentes eficaces en las áreas en las que enseñan. Los estándares bien escritos se asientan en una clara comprensión de lo que se considera un aprendizaje de calidad para los estudiantes. El Recuadro 1 ofrece un ejemplo de los estándares del Consejo Nacional para la Docencia de Matemáticas. Estos son accionados por una clara visión de la importancia de aprender matemáticas.

Recuadro 1: Un ejemplo de estándares para la docencia basados en una visión sobre el aprendizaje de calidad

Los Estándares Profesionales para la Docencia de Matemáticas (NCTM, 1989) del Consejo Nacional para la Docencia de Matemáticas fueron uno de los primeros estándares desarrollados por una asociación nacional de profesores.

Las normas del NCTM representan un ejemplo de estándares basados en una concepción claramente articulada del aprendizaje de calidad en un área temática específica, nacida gracias a la investigación profesional y académica. Fundamental para los estándares del NCTM fue el desarrollo de la facultad matemática para todos los estudiantes. Al respecto, podemos señalar que esta:

incluye la capacidad de explorar, hacer conjeturas y razonar en forma lógica; de resolver problemas no rutinarios; de comunicar acerca y a través de las matemáticas; y de conectar ideas dentro de las matemáticas y entre las matemáticas y otra actividad intelectual. La facultad matemática involucra, además, el desarrollo de la confianza personal y de la disposición a buscar, evaluar y usar información cuantitativa y espacial en la solución de problemas y en la toma de decisiones. La flexibilidad, la perseverancia, el interés, la curiosidad y la inventiva de los estudiantes también afectan el logro de la facultad matemática (p. 1).

Los profesores que redactaron los estándares del NCTM comenzaron con esta visión de aprendizaje de calidad, la que se convirtió en la base de los procesos que continuaron. El paso siguiente fue describir el tipo de docencia que podría producir ese aprendizaje, para finalmente concluir con un análisis del conocimiento, las habilidades y las disposiciones que necesitaba un profesor para entregar ese tipo de docencia, apoyado cuando fuera posible por la investigación sobre docencia y el aprendizaje de matemáticas.

Los estándares del NCTM intentan capturar aquello a lo que deberían aspirar los profesores titulados de matemáticas y, además, entregan metas desafiantes y a largo plazo para el aprendizaje profesional.

Los estándares para la docencia deben basarse en una visión coherente de lo que la sociedad considera una educación sólida y en la función de un profesor en el contexto de la entrega de esa 
educación. De acuerdo con esta comprensión, el equipo a cargo de la elaboración de estándares debiera evitar dos de los problemas característicos de los enfoques pasados a propósito de los criterios para evaluar a un profesor. El primero de ellos es ser demasiado amplios y generales al momento de evaluar, característica propia del tipo de evaluación usada en el siglo pasado, la cual se centraba en los aspectos de la personalidad (Kennedy, 2010). En tanto que el segundo de los problemas es ser demasiado específicos, es decir, desglosar la docencia en un conjunto de competencias y enfocarse en la evaluación de comportamientos concretos y estrechos, lo cual tiene como consecuencia la pérdida del significado de lo que estaban intentando lograr los profesores.

\subsection{Niveles de afirmaciones dentro de marcos estándares}

A medida que avanzan en su tarea, los redactores de estándares describen principalmente el alcance del trabajo profesional y las responsabilidades de los profesores, para lo cual necesitan llegar a acuerdos acerca de los deberes y responsabilidades que tendrán estos y los principios básicos que los guiarán. Las afirmaciones que desarrollan en este sentido suelen moverse desde lo general a lo específico, como muestran los niveles en la Figura 1, es decir, transitan desde un principio hacia una descripción más detallada de lo que saben y hacen los buenos profesores y cuáles son sus elaboraciones para cada una de estas áreas de docencia.

Figura 1: Niveles de afirmaciones dentro de marcos estándares

\begin{tabular}{|l|l|}
\hline Nivel 1: Principios & Guían la visión del aprendizaje de calidad y la labor de los profesores. \\
\hline Nivel 2: Dominios & Organizan categorías para los estándares requeridos para la docencia. \\
\hline Nivel 3: Estándares & $\begin{array}{l}\text { Descripciones de lo que deberían saber y hacer los profesores dentro de cada } \\
\text { dominio. }\end{array}$ \\
\hline Nivel 4: Elaboraciones & $\begin{array}{l}\text { Elaboraciones de lo que significan los estándares para las áreas particulares } \\
\text { de docencia (estas forman la base para diseñar las evaluaciones y las rúbricas } \\
\text { requeridas para revisar el desempeño). }\end{array}$ \\
\hline
\end{tabular}

a) Nivel 1 Principios: Las afirmaciones a este nivel son altamente generalizadas y abstractas. Buscan capturar los valores y los fines educacionales más profundos y a largo plazo que persiguen los profesores. No están diseñadas para evaluar el conocimiento 
profesional o el desempeño de un profesor ni para juzgar la calidad de un curso de formación docente.

b) Nivel 2 Dominios: Estas afirmaciones definen el alcance de la labor de los profesores y las principales categorías dentro de ese trabajo.

c) Nivel 3 Estándares: Las afirmaciones a este nivel identifican los estándares con cada uno de los dominios del nivel 2. Los estándares forman la base para el desarrollo de las evaluaciones y describen lo que necesitan saber y hacer los profesores en áreas particulares de la docencia.

d) Nivel 4 Elaboraciones: Las afirmaciones a este nivel explican el significado de cada uno de los estándares dentro de cada dominio. Las elaboraciones señalan elementos de acciones observables y adecuadas, pero no describen los métodos o los estilos de docencia específicos. Existen muchas formas de cumplir con el estándar y aunque no estandarizan la docencia, proporcionan una base para usar rúbricas al evaluar el desempeño de un profesor.

3.2. Dominios: organizar las categorías de estándares para la docencia

Mientras los equipos a cargo de la elaboración de estándares analizan los tipos de conocimiento, habilidades y compromisos que, por ejemplo, deberían haber obtenido los nuevos profesores durante el seguimiento de sus programas de formación docente, pronto experimentan un desafío común que es decidir cómo contener y organizar todas las ideas que están produciendo. En este sentido podemos decir que así como existen muchas formas de rebanar una torta, existen también muchas formas de organizar los marcos de estándares para los profesores, aun cuando siga siendo la misma «torta».

Ahora bien, aunque no podemos dar por sentado que una forma sea la mejor para organizar las categorías o los principales dominios de la labor de los profesores, una notable similitud surgió en las categorías y subcategorías que nacen a partir de los estándares desarrollados en diferentes países. En alguna medida, se trata de una reflexión del trabajo hecho durante los últimos veinticinco años en el 
análisis de las posibilidades de justificar las afirmaciones acerca de un conocimiento base para la profesión docente (por ejemplo, Shulman, 1987; Reynolds, 1989; Cohen y Ball, 1999).

Darling-Hammond y Bransford (2005) reúnen gran parte de este trabajo en su libro, el cual aborda tres preguntas en relación con lo que los profesores principiantes necesitan saber y poder hacer:

- ¿Qué tipo de conocimiento necesitan tener los profesores eficaces respecto de su materia y del proceso de aprendizaje y desarrollo de sus estudiantes?

- ¿Qué habilidades necesitan los profesores para procurar experiencias de aprendizaje productivas para un grupo diverso de estudiantes, ofreciendo opiniones informativas acerca de las ideas de los estudiantes y evaluando de modo crítico las propias prácticas docentes para mejorarlas?

- ¿Qué compromisos profesionales hacen los profesores para ayudar a cada estudiante a tener éxito y continuar desarrollando sus propios conocimientos y habilidades, como individuos y como miembros de una profesión colectiva?

Estas tres preguntas ofrecen un marco conceptual que ahora es común a distintos conjuntos de estándares en muchos países como Australia, Chile, Inglaterra, Nueva Zelanda, Escocia y EE.UU. Al respecto, la Figura 2 ilustra que los estándares para cada país tienen un marco básico similar a nivel de dominio.

En dicha figura cada país agrupa sus estándares en categorías o dominios similares a los grupos identificados anteriormente por Darling-Hammond y Bransford (2005). En alguna medida estas categorías reflejan el sentido común, pero también identifican áreas de investigación que entregan cada vez más evidencia acerca de la docencia y del aprendizaje que debería ser central en el diseño de programas de formación docente.

Aunque existe un consenso internacional emergente acerca de las características de los estándares exigidos para los nuevos profesores, se podría esperar que las consideraciones culturales locales 
afecten el carácter de los estándares para la docencia. Sin embargo, a partir de los ejemplos internacionales que aparecen hasta ahora, este no parece ser el caso, al menos no en términos de la estructura y contenido del marco de estándares. En alguna medida, quizás esto se deba a que los países están compartiendo sus estándares e intercambiando información, aunque también ello se deba a que los estándares, por definición, intentan estar libres de contexto, incluso dentro de los países.

Figura 2: Organización de categorías (dominios) para estándares en seis países

\begin{tabular}{|l|l|l|l|l|l|}
\hline Australia & Arabia Saudita & Inglaterra & $\begin{array}{l}\text { Nueva } \\
\text { Zelanda }\end{array}$ & Escocia & $\begin{array}{l}\text { EE.UU. } \\
\text { (InTASC) }\end{array}$ \\
\hline $\begin{array}{l}\text { Conocimiento } \\
\text { profesional }\end{array}$ & $\begin{array}{l}\text { Preparación para } \\
\text { el aprendizaje }\end{array}$ & $\begin{array}{l}\text { Conocimiento } \\
\text { profesional y } \\
\text { comprensión }\end{array}$ & $\begin{array}{l}\text { Conocimiento } \\
\text { profesional }\end{array}$ & $\begin{array}{l}\text { Conocimiento } \\
\text { profesional y } \\
\text { comprensión }\end{array}$ & $\begin{array}{l}\text { El que aprende } \\
\text { y el aprendizaje } \\
\text { El contenido }\end{array}$ \\
\hline $\begin{array}{l}\text { Práctica } \\
\text { profesional }\end{array}$ & $\begin{array}{l}\text { Promover y } \\
\text { apoyar el } \\
\text { aprendizaje }\end{array}$ & $\begin{array}{l}\text { Habilidades } \\
\text { profesionales }\end{array}$ & $\begin{array}{l}\text { Práctica } \\
\text { profesional }\end{array}$ & $\begin{array}{l}\text { Habilidades y } \\
\text { capacidades } \\
\text { profesionales }\end{array}$ & $\begin{array}{l}\text { Práctica en } \\
\text { instrucción }\end{array}$ \\
\hline $\begin{array}{l}\text { Compromiso } \\
\text { profesional }\end{array}$ & $\begin{array}{l}\text { Responsabilidades } \\
\text { profesionales }\end{array}$ & $\begin{array}{l}\text { Atributos } \\
\text { profesionales }\end{array}$ & $\begin{array}{l}\text { Relaciones } \\
\text { y valores } \\
\text { profesionales }\end{array}$ & $\begin{array}{l}\text { Valores } \\
\text { profesionales } \\
\text { y compromiso } \\
\text { personal }\end{array}$ & $\begin{array}{l}\text { Compromiso } \\
\text { profesional } \\
\text { Responsabilidad } \\
\text { profesional }\end{array}$ \\
\hline
\end{tabular}

En un sentido, no es de sorprenderse que los dominios de estándares y su organización se vean tan similares entre distintos países. Sin duda, el conocimiento local cumplirá una función vital en la implementación de los estándares, pero articular este conocimiento de acuerdo con cada contexto escolar no es función de los estándares, ni tampoco lo es el que los estándares bien elaborados generen algún tipo de homogeneización. Es probable que la naturaleza de la labor de los profesores y los conceptos de buena docencia no sean una función del país o de la cultura en un grado importante. Por ejemplo, es poco probable que lo que necesita saber y hacer un profesor de matemáticas varíe mucho de un país a otro.

Junjun Chen (2010) exploró el concepto de docencia de excelencia en escuelas medias de China y encontró pocas variaciones respecto de los estándares de países occidentales. Del mismo modo, Irving (2005) concluyó que los profesores de matemáticas de Nueva Zelanda tenían conceptos prácticamente idénticos del logro en la 
docencia de matemáticas que los profesores de EE.UU. e Ingvarson y Kleinhenz (2006) llegaron a la misma conclusión para los profesores de ciencias en Australia y EE.UU.; lo anterior significa que cada grupo tenía conceptos muy similares de lo que necesitan saber y hacer los buenos profesores de ciencias. Para el caso de Chile, a principios de la década de 2000, el Ministerio de Educación decidió adoptar, con muy pocas variaciones, un conjunto de estándares para profesores principiantes de EE.UU.

\subsection{Estándares dentro de los dominios}

El paso siguiente para el equipo a cargo de la elaboración de estándares es identificar aquello que se incluirá en cada uno de los dominios. La Figura 3 muestra un conjunto común de dominios y estándares de Australia, los que se agruparon en siete estándares para profesores egresados bajo tres dominios más amplios identificados por DarlingHammond y Bransford (2005).

Este marco de estándares es similar al de muchos otros países $\mathrm{y}$, de hecho, en el ámbito internacional, la mayoría de los conjuntos de estándares para profesores de aula son similares, organizándose alrededor de tres o cuatro categorías o dominios principales como se muestra en la Figura 1. Cada uno contiene estándares similares, por ejemplo, conocimiento de materias y cómo los estudiantes las aprenden, creación de un entorno de aprendizaje favorable o la contribución a la comunidad profesional de la escuela.

Figura 3: Estándares profesionales nacionales para el marco de docencia en Australia

\begin{tabular}{|c|c|}
\hline Dominios & Estándares dentro de cada dominio \\
\hline \multirow{2}{*}{$\begin{array}{l}\text { Conocimiento } \\
\text { profesional }\end{array}$} & Conocer a los estudiantes y cómo aprenden. \\
\hline & Conocer el contenido y cómo enseñarlo. \\
\hline \multirow{3}{*}{ Práctica profesional } & Planificar e implementar el aprendizaje y la docencia eficaces. \\
\hline & Evaluar, entregar opiniones e informar acerca del aprendizaje del estudiante. \\
\hline & Crear y mantener entornos de aprendizaje favorables y seguros. \\
\hline \multirow{2}{*}{$\begin{array}{l}\text { Responsabilidades } \\
\text { profesionales }\end{array}$} & Comprometerse con el aprendizaje profesional. \\
\hline & Comprometerse profesionalmente con colegas, padres y la comunidad. \\
\hline
\end{tabular}

El objetivo de estos marcos es fijar las principales dimensiones del conocimiento y la práctica de los profesores en forma clara y lógica dentro de áreas específicas que definen una base de 
conocimiento y sirven de mapa para el aprendizaje profesional de los profesores.

El significado y alcance de cada uno de los estándares en el marco australiano se elabora mediante la creación de una lista de elementos o indicadores. La Figura 4 muestra seis indicadores para cada uno de los dos estándares en el dominio del conocimiento profesional.

Figura 4: Dominio 1: Estándares profesionales nacionales para el marco de docencia en Australia:

\begin{tabular}{|c|c|c|}
\hline Dominio & Estándar & Indicadores \\
\hline \multirow[t]{2}{*}{$\begin{array}{l}\text { Conocimiento } \\
\text { profesional }\end{array}$} & $\begin{array}{l}\text { 1. Conocer } \\
\text { a los } \\
\text { estudiantes } \\
\text { y cómo } \\
\text { aprenden. }\end{array}$ & $\begin{array}{l}\text { 1.1. Desarrollo y características físicas, sociales e intelectuales de los } \\
\text { estudiantes. } \\
\text { 1.2. Comprender cómo aprenden los estudiantes. } \\
\text { 1.3. Estudiantes con diferentes antecedentes lingúísticos, culturales, } \\
\text { religiosos y socioeconómicos. } \\
\text { 1.4. Estrategias para enseñar a estudiantes aborígenes e isleños del } \\
\text { estrecho de Torres. } \\
\text { 1.5. Docencia diferenciada para satisfacer necesidades de aprendizaje } \\
\text { especiales de estudiantes en todo el rango de capacidades. } \\
\text { 1.6. Estrategias para apoyar la plena participación de estudiantes con } \\
\text { discapacidad. }\end{array}$ \\
\hline & $\begin{array}{l}\text { 2. Conocer } \\
\text { el contenido } \\
\text { y cómo } \\
\text { enseñarlo. }\end{array}$ & $\begin{array}{l}\text { 2.1. Estrategias de contenido y docencia del área de docencia. } \\
\text { 2.2. Selección y organización de contenido. } \\
\text { 2.3. Currículo, evaluación e información. } \\
\text { 2.4. Comprender y respetar a los habitantes aborígenes e isleños } \\
\text { del estrecho de Torres a fin de promover la reconciliación entre } \\
\text { australianos indígenas y no indígenas. } \\
\text { 2.5. Estrategias de alfabetismo y matemáticas. } \\
\text { 2.6. Tecnología de la información y las comunicaciones (TIC). }\end{array}$ \\
\hline
\end{tabular}

Cada uno de los estándares e indicadores se describen con más detalle en cuatro niveles de certificación, como se muestra en la Figura 5: profesor egresado, profesor competente, profesor con altos logros y profesor líder ${ }^{3}$.

3 Una lista completa de indicadores para todos los estándares del marco australiano se encuentra en el sitio web AITSL (http://www.teacherstandars. aitsl.edu.au/) 
Figura 5: Estándar 1: Estándares profesionales nacionales para el marco de docencia de Australia

\begin{tabular}{|c|c|c|c|c|c|}
\hline \multirow{2}{*}{\multicolumn{2}{|c|}{$\begin{array}{l}\text { DOMINIO } 1 \\
\text { ESTÁNDAR } 1\end{array}$}} & \multicolumn{4}{|c|}{ NIVELES DE CERTIFICACIÓN/ETAPAS DE CARRERAS } \\
\hline & & $\begin{array}{l}\text { Profesor } \\
\text { egresado }\end{array}$ & $\begin{array}{c}\text { Profesor } \\
\text { competente }\end{array}$ & $\begin{array}{c}\text { Profesor con } \\
\text { altos logros }\end{array}$ & $\begin{array}{c}\text { Profesor } \\
\text { líder }\end{array}$ \\
\hline $\begin{array}{l}\text { CONOCIMIENTO } \\
\text { PROFESIONAL } \\
\text { 1. Conocer a los } \\
\text { estudiantes y cómo } \\
\text { aprenden. }\end{array}$ & \begin{tabular}{|l|}
1.1 \\
Desarrollo y \\
características \\
físicas, sociales \\
e intelectuales \\
de los \\
estudiantes.
\end{tabular} & $\begin{array}{l}\text { Demostrar } \\
\text { conocimiento } \\
\text { y } \\
\text { comprensión } \\
\text { del desarrollo } \\
\text { y las } \\
\text { características } \\
\text { físicas, } \\
\text { sociales e } \\
\text { intelectuales } \\
\text { de los } \\
\text { estudiantes } \\
\text { y cómo } \\
\text { afectan el } \\
\text { aprendizaje. }\end{array}$ & \begin{tabular}{|l|} 
Usar \\
estrategias \\
docentes \\
basadas en el \\
conocimiento \\
del desarrollo \\
y las \\
características \\
físicas, \\
sociales e \\
intelectuales \\
de los \\
estudiantes a \\
fin de mejorar \\
el aprendizaje \\
de los \\
estudiantes.
\end{tabular} & $\begin{array}{l}\text { Seleccionar } \\
\text { un repertorio } \\
\text { flexible y } \\
\text { eficaz de } \\
\text { estrategias } \\
\text { docentes } \\
\text { para incluir } \\
\text { el desarrollo } \\
\text { y las } \\
\text { características } \\
\text { físicas, } \\
\text { sociales e } \\
\text { intelectuales } \\
\text { de los } \\
\text { estudiantes. }\end{array}$ & $\begin{array}{l}\text { Liderar a } \\
\text { colegas para } \\
\text { que seleccionen } \\
\text { y desarrollen } \\
\text { estrategias } \\
\text { de docencia } \\
\text { para mejorar } \\
\text { el aprendizaje } \\
\text { del estudiante, } \\
\text { usando el } \\
\text { conocimiento } \\
\text { del desarrollo y } \\
\text { las características } \\
\text { físicas, sociales e } \\
\text { intelectuales de } \\
\text { los estudiantes. }\end{array}$ \\
\hline
\end{tabular}

En la actualidad se escriben más elaboraciones de los estándares australianos, junto con ejemplares para las diferentes áreas de contenido y áreas de docencia. Algunos redactores de estándares prefieren una lista de indicadores para elaborar el significado de los estándares; otros en cambio privilegian un estilo en prosa para escribir algo que intenta reflejar la naturaleza integrada de la buena docencia, con el fin de evitar que los estándares se usen como una simple lista de elementos en una plantilla de evaluación. Volveremos sobre este tema más adelante en el análisis de ejemplos de estándares específicos del contenido.

\section{Estándares genéricos}

El australiano es un ejemplo de un marco genérico de estándares, en el sentido de que suele ser común a la mayoría de los profesores, sin importar el nivel en el que estos enseñan o los contenidos que enseñan. Los marcos de Inglaterra, Nueva Zelanda, Escocia y EE.UU. (InTASC) también son genéricos y Singapur acaba de elaborar un conjunto de competencias para profesores en proceso de egreso ${ }^{4}$.

4 Documento completo disponible en: http://www.nie.edu.sg/files/about-nie/TE21\%20 online\%20version.pdf 
En el ámbito internacional, los marcos de estándares más actuales siguen siendo genéricos, con algunas excepciones importantes como aquellos desarrollados por Chile y el Directorio Nacional de Estándares Profesionales para la Docencia en EE.UU., que se analizarán posteriormente. Al igual que en el marco australiano, las afirmaciones dentro de estos parámetros se organizan en diferentes niveles, desde dominios amplios que establecen las principales categorías de la labor de los profesores. Los estándares y sus elaboraciones deberían indicar las acciones observables y adecuadas, incluyendo referencias a prácticas específicas.

Por otra parte, el uso lógico y cuidadoso del lenguaje es importante al momento de elaborar los estándares, ello con el fin de evitar la superposición y la repetición. Como se mencionó anteriormente, Darling-Hammond y Bransford (2005) hacen una distinción clara y lógica entre los tres dominios principales de conocimiento, habilidades y compromisos. El conocimiento de las materias, por ejemplo, está en el dominio 1. Sin embargo, la habilidad de ayudar a los estudiantes a aprender esa materia está en el dominio 2. Los dominios 1 y 2 son conceptualmente distintos: aunque el dominio 1 se trata de lo que necesita saber un profesor egresado con el fin de planificar y enseñar bien, el dominio 2 se trata del desempeño en la clase, con los estudiantes, describiendo aquello de lo que son capaces de hacer los buenos profesores para promover el aprendizaje de los alumnos.

Al desarrollar marcos de estándares es preferible diferenciar las afirmaciones acerca de lo que los profesores deberían saber, de aquellas afirmaciones acerca de las habilidades o del desempeño (esto es, lo que los profesores deberían ser capaces de hacer) y las afirmaciones acerca de los compromisos mayores de los profesores como miembros de una profesión. Lo anterior debido a que algunos marcos de estándares no logran hacer esta distinción.

La distinción entre el dominio 1 (conocimiento) y el dominio 2 (desempeño) es similar a la distinción entre el «saber qué» y el «saber cómo» hecha por Gilbert Ryle (1946). Los estándares australianos tienden a desdibujar esta distinción refiriéndose, por ejemplo, a estrategias de docencia «sabiendo cómo enseñar el contenido del 
dominio $1 »$, cuando las habilidades como estas son más adecuadas puestas en el dominio 2 .

La incapacidad de hacer esta distinción puede originar una repetición innecesaria en un conjunto de estándares e indicadores. Los siguientes ejemplos de estándares genéricos se usarán para ilustrar esta idea.

También es importante garantizar que las afirmaciones en un marco de estándares se encuentren en el «nivel» adecuado en el marco (es decir, en el nivel de dominio, estándar o indicador), así como también lo estén los estándares.

\subsection{Ejemplos de marco genérico de estándares}

\subsubsection{El marco para la docencia de Danielson}

El marco para la docencia desarrollado por Charlotte Danielson ofrece un ejemplo de un marco de estándares genérico en donde los dominios se distinguen claramente y existe una repetición mínima. La Figura 6 proporciona un resumen de ello. En dicha estructura se muestra la profunda reflexión que se aplicó en la planificación, así como su lógica coherente, además de evidenciar cómo se han hecho claras distinciones entre los estándares de conocimiento en el dominio 1, los estándares de desempeño en los dominios 2 y 3 y las responsabilidades profesionales más amplias en el dominio 4. El marco detallado ofrece rúbricas para evaluar a los profesores en cada estándar en los cuatro niveles, esto es, nivel insatisfactorio, básico, muy competente y destacado. 
Figura 6: Marco para la docencia de Danielson

\begin{tabular}{|c|c|}
\hline Dominios & Estándares \\
\hline 1: Planificación y preparación & $\begin{array}{l}\text { a) Demostrar conocimiento de contenidos y pedagogía. } \\
\text { b) Demostrar conocimiento de los estudiantes. } \\
\text { c) Seleccionar metas educacionales. } \\
\text { d) Demostrar conocimiento de los recursos. } \\
\text { e) Diseñar instrucciones coherentes. } \\
\text { f) Diseñar evaluaciones para los estudiantes. }\end{array}$ \\
\hline 2: En entorno del aula & $\begin{array}{l}\text { a) Crear un entorno de respeto y compenetración. } \\
\text { b) Establecer una cultura del aprendizaje. } \\
\text { c) Manejar los procedimientos del aula. } \\
\text { d) Manejar el comportamiento de los estudiantes. } \\
\text { e) Organizar el espacio físico. }\end{array}$ \\
\hline 3: Instrucción & $\begin{array}{l}\text { a) Comunicarse con los estudiantes. } \\
\text { b) Usar técnicas de preguntas y análisis. } \\
\text { c) Comprometer a los estudiantes en el aprendizaje. } \\
\text { d) Usar evaluaciones en la instrucción. } \\
\text { e) Demostrar flexibilidad y capacidad de respuesta. }\end{array}$ \\
\hline 4: Responsabilidades profesionales & $\begin{array}{l}\text { a) Reflexionar en torno a la docencia. } \\
\text { b) Mantener registros exactos. } \\
\text { c) Comunicarse con las familias. } \\
\text { d) Participar en una comunidad profesional. } \\
\text { e) Crecer y desarrollarse profesionalmente. } \\
\text { f) Mostrar profesionalismo. }\end{array}$ \\
\hline
\end{tabular}

El marco de Danielson fue diseñado como un instrumento para la observación en el aula, con entrevistas antes y después de las lecciones acerca de las mismas y es el instrumento más utilizado en EE.UU. para la evaluación de profesores. Esta herramienta también es notable debido a que cuenta con una dinámica básica clara en cuanto a su estructura, la que es representativa del ciclo común del trabajo de los profesores, incluyendo desde la planificación para enseñar, hasta la evaluación y la reflexión para preparar la próxima lección. Así mismo, reconoce la importancia de entrevistar a los profesores antes y después de la observación con el fin de tener una comprensión clara respecto del razonamiento detrás de sus acciones y de su capacidad para analizar los efectos de las mismas en el aprendizaje de los estudiantes. Sin embargo, aunque el marco de Danielson es una base muy útil para evaluar el desempeño de los profesores en el aula, no fue diseñado como una guía más general del contenido de los programas de formación docente, a diferencia del ejemplo siguiente. 
4.1.2. Los estándares para la docencia del modelo del InTASC El Consorcio Interestatal de Apoyo y Evaluación de Profesores (InTASC, por sus siglas en inglés) es un consorcio estatal de organismos de educación y organizaciones nacionales de educación en EE.UU., dedicado a aplicar reformas a la preparación, certificación y desarrollo profesional constante de los profesores. Los organismos que forman parte del consorcio se reunieron por primera vez en 1992 con la finalidad de crear un conjunto de estándares que articularan el núcleo común del conocimiento y las habilidades de docencia que deberían tener todos los nuevos profesores. El «núcleo común» trazaba «las bases y principios comunes de la práctica docente que atraviesan todas las áreas temáticas y niveles y que todos los profesores comparten» (InTASC, 2010: 4).

En 2010, InTASC publicó una versión revisada de sus estándares modelo desarrollados en el año 1992, instancia en la cual quedó en evidencia el interesante contraste en relación con los estándares analizados anteriormente y los nuevos, debido a la forma en la que aquellos se organizaron.

La Figura 7 muestra que el primer marco para los estándares InTASC se dividía en cuatro dominios. Mientras que los nuevos estándares comprenden un conjunto de diez principios de docencia eficaz, los que han sido revisados para poder dar respuesta a las nuevas expectativas para la docencia. Así mismo, dichos principios tienen la finalidad de ayudar a las autoridades responsables de la formulación de políticas y a los educadores a lograr sus metas de reforma en educación y están diseñados para ser compatibles con los estándares de núcleo común (currículo) recientemente publicados para EE.UU. y con otros estándares para el caso de la educación profesional.

La cita siguiente ilustra esta postura, la que fue tomada de la introducción al núcleo modelo de estándares para la docencia que desarrolló el Consorcio Interestatal de Apoyo y Evaluación de Profesores (InTASC) del Council of Chief State School Officers (CCSSO).

El siguiente «núcleo modelo de estándares para la docencia» es un esfuerzo inicial para articular a través de la lente del profesor, lo que serían la docencia y el 
aprendizaje eficaces en ese nuevo sistema. El comité encargado de elaborar el borrador comenzó centrándose en el aprendiz: ¿quiénes son nuestros estudiantes de hoy? ¿qué activos y necesidades aportan a la experiencia de aprendizaje? ¿qué los compromete y cómo abordan la expectativa de lo que cada estudiante aprenderá en un nivel superior? Es claro que los estudiantes de hoy son más diversos racial y lingüísticamente, y tienen necesidades especiales. Aun así, sus diferencias son fortalezas. (InTASC, 2010)

Figura 7: Núcleo modelo de estándares para la docencia del InTASC (versión preliminar)

\begin{tabular}{|c|c|}
\hline Dominios & Estándares \\
\hline \begin{tabular}{|l} 
El estudiante y \\
el aprendizaje
\end{tabular} & $\begin{array}{l}\text { 1. Desarrollo del estudiante: El profesor comprende cómo aprenden y se desarrollan } \\
\text { los niños, reconociendo que los patrones de aprendizaje y desarrollo varían en cada } \\
\text { individuo dentro y a través de las áreas cognitiva, lingüística, social, emocional y física } \\
\text { y diseña e implementa experiencias de aprendizaje desafiantes y adecuadas para ello. } \\
\text { 2. Diferencias de aprendizaje: El profesor usa la comprensión de las diferencias } \\
\text { individuales y la diversidad de comunidades para garantizar entornos de aprendizaje } \\
\text { inclusivos que permitan que cada estudiante alcance su pleno potencial. } \\
\text { 3. Entornos de aprendizaje: El profesor trabaja con los estudiantes para crear entornos } \\
\text { que apoyen el aprendizaje individual y colaborativo, fomentando la interacción social } \\
\text { positiva, el compromiso activo en el aprendizaje y la automotivación. }\end{array}$ \\
\hline $\begin{array}{l}\text { Conocimiento } \\
\text { del contenido }\end{array}$ & $\begin{array}{l}\text { 4. Conocimiento del contenido: El profesor comprende los conceptos centrales, las } \\
\text { herramientas de consulta y las estructuras de la o las disciplinas que enseña y crea } \\
\text { experiencias de aprendizaje que hacen que estos aspectos de la disciplina sean } \\
\text { accesibles y significativos para los estudiantes. } \\
\text { 5. Aplicaciones innovadoras del contenido: El profesor comprende cómo conectar } \\
\text { conceptos y usar diferentes perspectivas para comprometer a los estudiantes en } \\
\text { pensamiento crítico/creativo y en la solución colaborativa de problemas genuinos } \\
\text { relacionados con temas locales y mundiales. }\end{array}$ \\
\hline $\begin{array}{l}\text { Práctica de } \\
\text { instrucción }\end{array}$ & $\begin{array}{l}\text { 6. Evaluación: El profesor comprende y usa múltiples métodos de evaluación para } \\
\text { comprometer a los estudiantes en su propio crecimiento, documentar su avance y } \\
\text { nutrir la planificación e instrucción constante del profesor. } \\
\text { 7. Planificación para la instrucción: El profesor extrae conocimiento de áreas de } \\
\text { contenido, habilidades transdisciplinarias, de los estudiantes, la comunidad y la } \\
\text { pedagogía para planificar una instrucción que apoye a cada alumno en el logro de } \\
\text { rigurosas metas de aprendizaje. } \\
\text { 8. Estrategias de instrucción: El profesor comprende y usa una variedad de estrategias de } \\
\text { instrucción para incentivar a los estudiantes a desarrollar una profunda comprensión } \\
\text { de las áreas de contenido y sus conexiones y formar habilidades para tener acceso a } \\
\text { información y aplicarla en forma adecuada. }\end{array}$ \\
\hline $\begin{array}{l}\text { Responsabilidad } \\
\text { profesional }\end{array}$ & $\begin{array}{l}\text { 9. Reflexión y crecimiento continuos: El profesor es un profesional reflexivo que usa la } \\
\text { evidencia para evaluar continuamente su práctica, en particular, los efectos de sus elecciones } \\
\text { y acciones en los demás (estudiantes, familias y otros profesionales en la comunidad } \\
\text { de aprendizaje) y adapta su práctica para satisfacer las necesidades de cada estudiante. } \\
\text { 10. Colaboración: El profesor colabora con estudiantes, familias, colegas, otros } \\
\text { profesionales y miembros de la comunidad para compartir la responsabilidad por el } \\
\text { crecimiento y desarrollo, aprendizaje y bienestar del estudiante. }\end{array}$ \\
\hline
\end{tabular}


Cada uno de los diez estándares del InTASC tiene la estructura que se muestra en la Figura 8 para el caso del estándar 1. Estos estándares se presentan en una forma distinta de la mayoría: cada estándar es esencialmente un estándar de desempeño, ya que pone primero el desempeño que se espera de un profesor, luego los tipos de prácticas que se deberían observar y, por último, la evaluación en relación con el aprendizaje de los estudiantes. En este sentido no solo menciona el desempeño esperado, sino que también el conocimiento y los elementos de actitud (disposiciones) que apoyan o subrayan la capacidad de cumplir con ese estándar, identificando bajo las disposiciones fundamentales las actitudes y compromisos que conducen esas prácticas.

Figura 8: Estándar 1 del núcleo modelo de estándares para la docencia del InTASC

\begin{tabular}{|c|c|}
\hline \multicolumn{2}{|c|}{$\begin{array}{l}\text { Estándar 1: Desarrollo del estudiante } \\
\text { El profesor comprende cómo aprenden y se desarrollan los niños, reconociendo que sus patrones } \\
\text { de aprendizaje y desarrollo varían de un individuo a otro dentro y a través de las áreas cognitiva, } \\
\text { ingüística, social, emocional y física y diseña e implementa experiencias de aprendizaje desafiantes y } \\
\text { decuadas para su desarrollo. }\end{array}$} \\
\hline $\begin{array}{l}\text { tantemente el } \\
\text { a fin de diseñar } \\
\text { a satisfacer las } \\
\text { s en cada área } \\
\text { úística, social, } \\
\text { cia el siguiente } \\
\text { cción adecuada } \\
\text { ra las fortalezas } \\
\text { sus intereses y } \\
\text { cada estudiante } \\
\text { milias, colegas }\end{array}$ & $\begin{array}{l}\text { Conocimiento esencial } \\
\text { a) El profesor comprend } \\
\text { aprendizaje, esto es, cóm } \\
\text { conocimiento, adquieren ha } \\
\text { procesos de pensamiento dis } \\
\text { usar estrategias de instrucc } \\
\text { aprendizaje. } \\
\text { b) El profesor comprende qu } \\
\text { lingüístico, social, emociona } \\
\text { influye en el aprendizaje y sab } \\
\text { educacionales que considerer } \\
\text { c) El profesor identifica l } \\
\text { aprendizaje y comprende cóm } \\
\text { área pueden afectar el desem } \\
\text { d) El profesor comprende la } \\
\text { lalturen el }\end{array}$ \\
\hline \multicolumn{2}{|l|}{ Disposiciones fundamentales } \\
\hline \multicolumn{2}{|c|}{$\begin{array}{l}\text { a) El profesor respeta las diferentes fortalezas y debilidades de los estudiantes y se compromete a usar } \\
\text { esta información para el mejor desarrollo de cada uno. } \\
\text { b) El profesor se compromete a usar las fortalezas de los estudiantes como una base para el crecimiento } \\
\text { y sus ideas equivocadas, como oportunidades para el aprendizaje. } \\
\text { c) El profesor asume la responsabilidad de promover el crecimiento y desarrollo de los estudiantes. } \\
\text { d) El profesor valora el aporte y la contribución de las familias, colegas y otros profesionales en la } \\
\text { comprensión del desarrollo de cada estudiante. }\end{array}$} \\
\hline
\end{tabular}


Es necesario recordar también que el núcleo modelo de estándares para la docencia del InTASC proporciona el marco para elaboraciones más detalladas de estándares dentro de cada una de las áreas especializadas de la docencia, como por ejemplo la docencia primaria y la docencia científica secundaria. A diferencia del marco de Danielson, el propósito de los estándares del InTASC es entregar un currículo para programas de formación docente y cubre en mayor detalle lo que deberían ser los objetivos de un programa de este tipo.

Los del InTASC representan uno de los mejores ejemplos de estándares genéricos existentes y sin duda constituyen todo un desafío. No obstante, una preocupación que expresan los comentaristas especializados es que quizás sean demasiado complejos y fijen la vara en un nivel poco realista para los profesores principiantes.

Si bien los estándares del InTASC son dignos de admiración, otro inconveniente es el grado de repetición que este método de organización genera, a diferencia del marco de Danielson. En este sentido lo anterior se aplica especialmente al caso bajo el encabezado «Desempeño»: varios estándares, además del uno, mencionan elementos similares a los puntos a), b) y (c) de la columna sobre desempeño en la Figura 8. El estándar 1 se menciona en la mayoría de los otros estándares de una forma similar. Por ejemplo, afirmaciones como «planificación y docencia en formas que satisfagan las necesidades de cada uno de los estudiantes» se repite en varios estándares, dando con ello importancia gravitante al conocimiento de la materia. Algunas disposiciones, como respetar la diversidad del estudiante y valorar la colaboración también se mencionan en varios de ellos.

\section{Estándares para la docencia específicos al contenido y al nivel}

Ni el marco de Danielson ni los estándares InTASC pretenden analizar a fondo lo que significa cada estándar para las diferentes áreas de docencia, como la docencia primaria o aquella en materias de secundaria. Es claro que lo que un futuro profesor de primaria debería saber acerca de las características de sus estudiantes y los contenidos que enseña es distinto de lo que debería saber un profesor de secundaria. 
Un marco de estándares que permanece a nivel genérico ofrece una base limitada para evaluar el conocimiento y la práctica de los profesores. Por ejemplo, la mayoría de los estándares incluye una afirmación similar a «los profesores deberían comprender profundamente el contenido que se espera que enseñen». Es necesario explicar, entonces, el significado de esta afirmación en términos prácticos antes de que este estándar se convierta en una guía útil para desarrollar evaluaciones, puesto que lo que significa para un futuro profesor de matemáticas de primaria claramente será diferente de la profundidad de conocimiento que se espera para un futuro profesor de matemáticas de los cursos más altos de la secundaria.

En un sentido, todos los profesores son profesores especializados $y$, por ende, los estándares para la docencia necesitan identificar no solo lo que es común a todos ellos, sino también aquello que es único respecto de la buena docencia en las diferentes áreas especializadas de esta. Esto es cierto tanto para los profesores de primaria como para los de secundaria: los primeros son tan especializados en su área como los segundos, pero lo que necesita saber un profesor de la primera infancia respecto de cómo aprender a leer es muy distinto de lo que necesita saber un profesor de ciencias de secundaria acerca de ayudar a los estudiantes a aprender física; del mismo modo, lo que necesita saber un profesor de primaria respecto del desarrollo del niño es distinto de lo que necesita saber un profesor de secundaria en relación con el desarrollo del adolescente y así sucesivamente.

Lo anterior implica pues que es necesario elaborar estándares para dos niveles. Primero en términos genéricos, donde identificar aspectos del conocimiento y la práctica de los profesores que se aplican a todos ellos, sin importar qué o a quién enseñen. Los estándares núcleo de InTASC son un ejemplo de esta idea; en segundo término, los redactores de estándares necesitan moverse a niveles más profundos, donde elaboren lo que saben y hacen los buenos profesores en muchas de las áreas de especialización que conforman la profesión de docente.

Los estándares InTASC, por ejemplo, fueron elaborados considerando diversos ámbitos de especialización, los cuales son elegidos por cada país y dependen de la forma en la que se organiza 
la escolaridad dentro de él, aunque generalmente incluyen niveles como la educación de la primera infancia, la docencia primaria y la secundaria y, dentro de estos niveles, se definen las áreas de docencia en materias especializadas, como por ejemplo, matemáticas, lenguaje o arte, por ejemplo.

Como se aprecia, si los estándares van a ser útiles como guía para quienes desarrollan programas de formación docente, requieren ser «analizados más en profundidad» y ser más específicos acerca de lo que necesitan saber y hacer los profesores. Por ejemplo, los estándares genéricos necesitan ser elaborados de modo tal que identifiquen lo que es único dentro de lo que los profesores necesitan aprender en sus programas de formación docente (inglés, historia, matemáticas y ciencias, entre otros), diferenciando lo que saben y hacen los buenos profesores en las diferentes áreas de especialización. La Figura 9 grafica este análisis en profundidad para una sola área temática de matemáticas, los números.

Figura 9: Análisis en profundidad del nivel específico a la materia

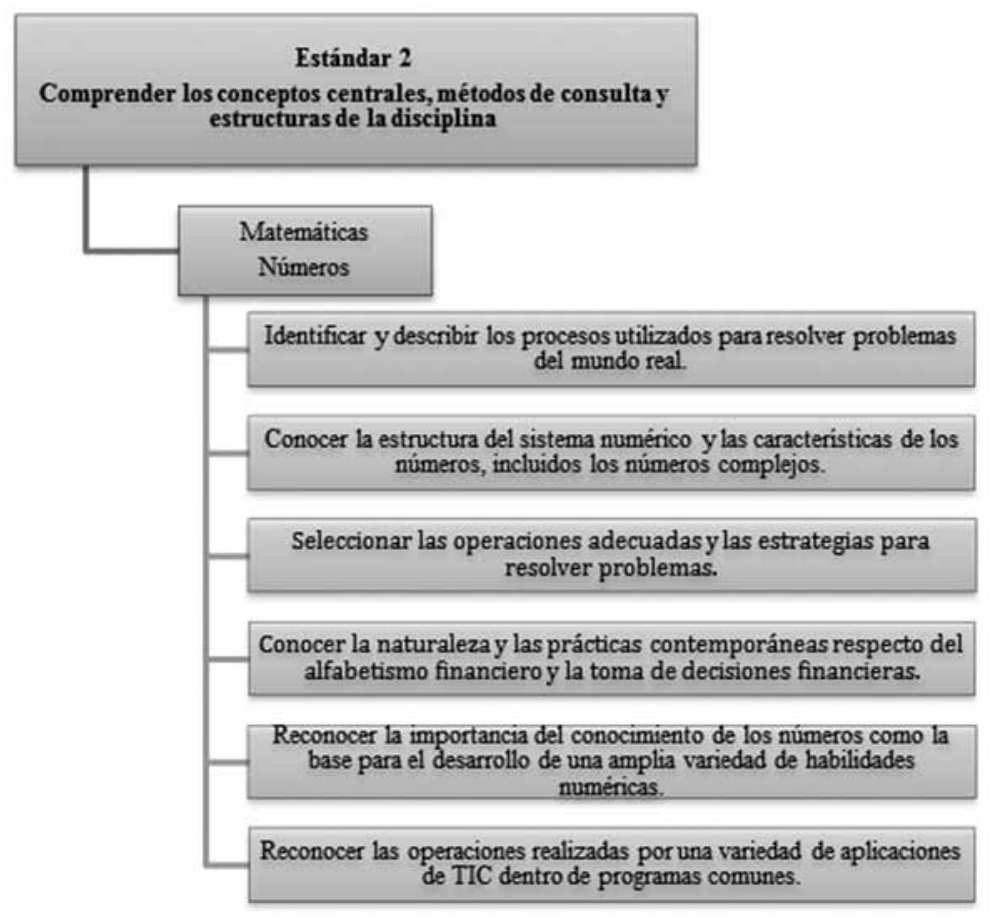


Los estándares para profesores primarios también deberían ofrecer una guía clara acerca de esas materias si van a ser de utilidad para la planificación y evaluación de programas. Por ejemplo, ¿qué debería saber un profesor de primaria respecto de las nuevas investigaciones en torno a aprender a leer? ¿qué estrategias deberían ser capaces de demostrar para superar las dificultades de lectura entre sus estudiantes?

\subsection{Ejemplos de estándares específicos al contenido}

La Junta Nacional para Estándares Profesionales para la Docencia (NBPTS, por sus siglas en inglés) en EE.UU. proporciona uno de los ejemplos mundiales más amplios de estándares específicos al contenido y al nivel. El NBPTS es una organización independiente, privada, imparcial y sin fines de lucro regida por un directorio, en donde la mayor parte de ella está conformada por profesores de aula. Su función principal es procurar un sistema de certificación profesional para profesores que las autoridades empleadoras puedan utilizar para recompensar a aquellos que cumplan con estándares altos. La certificación se basa en evaluaciones rigurosas acerca del conocimiento profesional y del desempeño de un profesor.

Desde su creación en 1987, cerca de 100.000 profesores han logrado la certificación de la Junta Nacional, aproximadamente la mitad de los que han postulado. Dicha certificación acaba de ser reconocida por el Consejo Nacional de Investigación por tener un impacto positivo en el logro académico, la retención de profesores y el desarrollo.

La Figura 10 entrega el ejemplo de un marco para estándares específicos al contenido de la NBPTS, en este caso para profesores secundarios de ciencias. Se trata de uno de los 25 conjuntos de estándares desarrollados por la Junta Nacional para la certificación de profesores titulados con altos logros para diferentes contenidos y niveles de escolaridad, lo que ha permitido que cerca del 95\% de los docentes haya tenido acceso a la respectiva certificación.

En la Figura 10 se aprecia que el conjunto completo de estándares de ciencias contiene elaboraciones de cada uno de los 13 estándares. Por ejemplo, el estándar 2 Conocimiento de ciencias analiza 
en detalle lo que los profesores deberían saber acerca de la naturaleza de la ciencia y de las ideas fundamentales en cada terreno científico ${ }^{5}$.

Por otro lado podemos mencionar que existen varias cosas por observar en relación con los estándares de la NBPTS expresados en la Figura 10. Lo primero es que estos tienen una estructura de dominio básica similar a los estándares genéricos descritos anteriormente, es decir, existen estándares que cubren el conocimiento, la práctica y las responsabilidades profesionales. Así mismo, al igual que el marco de Danielson, estos estándares se organizan en una forma que calza con la estructura del trabajo de los profesores, reflejando la naturaleza dinámica de lo que hacen los buenos profesores. El dominio 1 comienza con lo que saben y pueden hacer los buenos profesores para planificar y preparar el aprendizaje de los estudiantes; el dominio 2 incluye lo que hacen los buenos profesores para establecer un entorno favorable para el aprendizaje de la ciencia; el dominio 3 identifica aquello que es único en el tipo de aprendizaje que debería promover un buen profesor de ciencias; y el dominio 4 cubre las responsabilidades profesionales más amplias de un profesor de ciencias en la escuela y en la comunidad.

Estos tres estándares se basan en lo que los expertos en currículo de ciencias consideran los principales elementos del aprendizaje de calidad en la ciencia y forman los tres objetivos principales para enseñar ciencias. En segundo término, los estándares de la NBPTS identifican con mayor detalle los tipos de conocimiento que necesitan los profesores de ciencia y los tipos de aprendizaje que ellos debieran promover. A modo de ejemplo, en la introducción a los estándares en ciencia se indica que:

(...) los estándares están diseñados para especificar los aspectos fundamentales de la docencia científica de altos logros. En la práctica, las facetas de la docencia mencionadas en los estándares forman un tejido muy denso y suelen ocurrir en forma simultánea debido a que la docencia ejemplar es una actividad sin interrupciones con muchos objetivos dispares que se atienden en un momento determinado

5 Revisar documento completo en www.nbpts.org 
Figura 10: Estándares de la NBPTS para profesores titulados en ciencias en las escuelas secundarias

\begin{tabular}{|c|c|}
\hline Dominios & Estándares \\
\hline $\begin{array}{l}\text { I. Preparar } \\
\text { el camino } \\
\text { para el } \\
\text { aprendizaje } \\
\text { productivo } \\
\text { de los } \\
\text { estudiantes }\end{array}$ & $\begin{array}{l}\text { 1. Comprensión de los estudiantes: Los profesores de ciencias con altos logros saben cómo } \\
\text { aprenden los estudiantes, los conocen activamente como individuos y determinan su } \\
\text { comprensión de la ciencia al igual que su bagaje de aprendizaje individual. } \\
\text { 2. Conocimiento de ciencias: Los profesores de ciencias con altos logros tienen un } \\
\text { conocimiento de ciencias y de educación científica amplia y actual, junto con un profundo } \\
\text { conocimiento de cada una de las subáreas de la ciencia, lo que usan para fijar metas de } \\
\text { aprendizaje importantes. } \\
\text { 3. Recursos de instrucción: Los profesores de ciencias con altos logros seleccionan y adaptan } \\
\text { recursos de instrucción, lo que incluye los recursos de tecnología y laboratorio y de la } \\
\text { comunidad y crean recursos propios para apoyar la exploración activa de la ciencia por } \\
\text { parte del estudiante. }\end{array}$ \\
\hline $\begin{array}{l}\text { II. Establecer } \\
\text { un contexto } \\
\text { favorable } \\
\text { para el } \\
\text { aprendizaje } \\
\text { de los } \\
\text { estudiantes }\end{array}$ & $\begin{array}{l}\text { 4. Compromiso: Los profesores de ciencias con altos logros estimulan el interés en la } \\
\text { ciencia y la tecnología y obtienen la participación mantenida de sus estudiantes en las } \\
\text { actividades de aprendizaje. } \\
\text { 5. Entorno de aprendizaje: Los profesores de ciencias con altos logros crean entornos de } \\
\text { aprendizaje seguros y favorables que fomentan las altas expectativas para el éxito de todos } \\
\text { los estudiantes y en los que estos ponen los valores inherentes a la práctica de la ciencia. } \\
6 \text { Participación equitativa: Los profesores de ciencias con altos logros dan pasos para } \\
\text { garantizar que todos los estudiantes, incluidos los grupos que históricamente no se } \\
\text { sienten incentivados a ingresar al mundo de la ciencia, participen en el estudio de esta. }\end{array}$ \\
\hline $\begin{array}{l}\text { III. Progresar } \\
\text { en el } \\
\text { aprendizaje } \\
\text { de los } \\
\text { estudiantes }\end{array}$ & $\begin{array}{l}\text { 7. Indagación científica: Los profesores de ciencias con altos logros desarrollan en } \\
\text { los estudiantes las operaciones mentales, los hábitos de la mente y las actitudes que } \\
\text { caracterizan el proceso de indagación científica. } \\
\text { 8. Comprensión conceptual: Los profesores de ciencias con altos logros usan una variedad } \\
\text { de estrategias e instrucción para ampliar la comprensión de los estudiantes acerca de las } \\
\text { principales ideas de la ciencia. } \\
\text { 9. Contextos científicos: Los profesores de ciencias con altos logros crean oportunidades } \\
\text { para que los estudiantes analicen los contextos humanos de la ciencia, incluso su historia, } \\
\text { relaciones recíprocas con la tecnología, lazos con las matemáticas y el impacto en la } \\
\text { sociedad para que los estudiantes hagan conexiones entre disciplinas científicas y con } \\
\text { otras áreas de contenido. }\end{array}$ \\
\hline \begin{tabular}{|l|} 
IV. Apoyar \\
la enseñanza \\
y el \\
aprendizaje \\
de los \\
estudiantes
\end{tabular} & $\begin{array}{l}\text { 10. Evaluación: Los profesores de ciencias con altos logros evalúan el aprendizaje de } \\
\text { los estudiantes a través de una variedad de medios que se alinean con las metas de } \\
\text { aprendizaje establecidas. } \\
\text { 11. Alcance de la familia y la comunidad: Los profesores de ciencias con altos logros } \\
\text { trabajan en forma productiva con familias y comunidades a fin de servir a los mejores } \\
\text { intereses de cada estudiante. } \\
\text { 12. Compañerismo y liderazgo: Los profesores de ciencias con altos logros contribuyen } \\
\text { a mejorar la calidad de la práctica de sus colegas, en el programa de instrucción y en el } \\
\text { trabajo de la comunidad profesional más grande. } \\
\text { 13. Reflexión: Los profesores de ciencias con altos logros analizan, evalúan y fortalecen } \\
\text { en forma constante su práctica, con el fin de mejorar la calidad de las experiencias de } \\
\text { aprendizaje de sus estudiantes. }\end{array}$ \\
\hline
\end{tabular}

Una característica especial de los estándares de la NBPTS es el tercer dominio. En efecto, en primer término y a diferencia de muchos 
estándares genéricos, este dominio deja claro que se espera que los profesores titulados proporcionen evidencia de que pueden progresar en el aprendizaje de los estudiantes y también analiza profundamente a fin de identificar los tipos de aprendizaje que debería poder promover un profesor, en este caso un profesor de ciencias. Finalmente, se identifican tres tipos principales de aprendizaje: indagación científica, comprensión conceptual y contextos de la ciencia.

En segunda instancia los estándares dejan claro que se espera que un profesor que postula a la certificación de la Junta Nacional entregue evidencia de que puede promover cada tipo de aprendizaje entre sus estudiantes. En forma similar, los estándares desarrollados por la NBPTS para otras áreas temáticas y niveles de escolaridad identifican los tipos únicos de aprendizaje que se espera que promuevan los profesores en sus áreas especializadas.

En tercer lugar, aunque estos como conjunto sean específicos a la docencia de ciencias, existen varios estándares individuales que siguen siendo genéricos y similares en todos los estándares de la NBPTS e incluyen, por ejemplo, los estándares en el dominio 2 acerca del entorno de aprendizaje, la participación equitativa y los estándares 11 a 13 en el dominio 4 con respecto a las responsabilidades profesionales más amplias. El contenido de estos sigue siendo prácticamente el mismo en las diferentes áreas de especialización, sin embargo cada una todavía necesita una cuidadosa elaboración.

Finalmente, una función clave de los estándares de la NBPTS es que han sido desarrollados con sus fines de evaluación en mente, es decir, se crearon para que se convirtieran en una guía clara acerca de lo que se va a evaluar. Por ejemplo, es evidente que los estándares requieren de diferentes tipos de evaluación en donde algunos, como el estándar 2 requiere evaluaciones escritas que cubran el conocimiento de la materia que se espera de los profesores de ciencias; otros, como los estándares 1 y 10, también requieren evaluaciones del conocimiento de un profesor acerca de cómo ayudar a los estudiantes a aprender esa materia y cómo identificar y manejar las dificultades que puedan surgir (los profesores que postulan a la certificación de la Junta Nacional asisten a centros de evaluación designados en 
donde se evalúa su conocimiento por medio de ejercicios de respuesta construidos y efectuados en una computadora).

Los estándares 7,8 y 9 requieren un tipo distinto de evaluación, basada en la evidencia del desempeño de un profesor durante la entrega de tres instancias distintas para que los estudiantes logren su aprendizaje. Aquí es donde la NBPTS hizo un aporte importante a los métodos de evaluación de profesores, ya que compromete los servicios de expertos en evaluación educacional para desarrollar un portafolio de tareas estructuradas y basadas, por ejemplo, en muestras de la labor de los estudiantes durante el tiempo que demoren en alcanzar el aprendizaje, y cintas de video de la interacción de la clase que demuestren la capacidad de mantener una conversación de calidad. La NBPTS también creó portafolios de tareas diseñadas para evaluar estándares en el dominio 4, basadas en registros de contribución a la escuela y a la comunidad profesional.

Los profesores que postulan a la certificación de la Junta Nacional realizan un total de diez tareas de evaluación; seis ejercicios del centro de evaluaciones y cuatro ejercicios de portafolio. Juntos, estos ejercicios entregan evidencia para cada uno de los trece estándares contemplados. Los ejercicios, por su parte, son evaluados por profesores que trabajan en la misma área de docencia y que han sido capacitados con altos niveles de exigencia para cumplir su labor.

La longitud de los estándares específicos al contenido, como los de la NBPTS, representa una inquietud para algunos, ya que suelen ser más detallados que los estándares genéricos. En nuestra experiencia, por lo general los administradores están más preocupados de esto que los profesores y aunque la brevedad en algunos casos puede ser una virtud, el peligro con los conjuntos de estándares genéricos es que, en un análisis detallado, pueden desviar el significado y el propósito educacional, puesto que no reflejan bien la complejidad y la sofisticación de lo que saben y hacen los buenos profesores. Por lo tanto, carecen de la capacidad de inspirar, que es básicamente el significado original de un estándar. Debido a su generalidad, igualmente, tienen una capacidad limitada para guiar la formación docente o para medir y también es poco probable que ganen el respeto 
que los profesores tienen por los estándares desarrollados por los profesores para la NBPTS.

\subsection{Estándares para la docencia en Chile}

Chile representa un ejemplo de un país que ha hecho avances rápidos en el desarrollo de estándares para la docencia en los últimos diez años (Meckes, 2011). Lo que hace que este desarrollo sea altamente pertinente a otros países es la medida en la que Chile ha traspasado los estándares genéricos para la docencia, elaborando estándares específicos a profesores de primaria y a profesores especializados en escuelas secundarias.

En el año 2003, el Ministerio de Educación chileno publicó un Marco genérico de buena docencia, después de un amplio proceso de consulta con profesores y otros grupos de interés, el cual es ampliamente utilizado actualmente para la evaluación de profesores en ejercicio. Todos los profesores de escuelas públicas deben aprobar una evaluación cada cuatro años.

Ahora bien, durante la década del 2000, creció en Chile la inquietud respecto del desempeño de los estudiantes de este país en pruebas internacionales de logro académico como PISA y TIMSS. Ello, debido a que en 2004 un informe de la OCDE acerca de la educación chilena identificó que las debilidades en el sistema de formación docente podrían representar una de las razones de este bajo desempeño escolar, especialmente en lo referido al sistema para acreditar programas de formación docente. Así, tanto los mecanismos para garantizar la calidad del sistema de formación docente como la calidad de los profesores principiantes eran débiles, lo que se veía agravado por una oferta excesiva de egresados. El estudio de IEA TEDS-M mostró por ejemplo que los futuros profesores de matemáticas que egresaban de programas de formación docente tenían evaluaciones significativamente menores en pruebas de conocimiento matemático y conocimiento pedagógico en matemáticas que la mayoría de los futuros docentes de los países que participaban en el estudio (Ingvarson, et al., no publicado). 
Como resultado de estas inquietudes, en el año 2009 el Ministerio de Educación chileno encargó el desarrollo de estándares nacionales para profesores primarios egresados y el diseño de métodos para evaluarlos: era necesario elaborar preferentemente el marco genérico de buena docencia para que el esfuerzo resultara útil en la planificación del contenido de programas de formación docente y en la evaluación de egresados. De esta forma, dicho documento contenía afirmaciones tales como las siguientes:

- Los profesores dominan los contenidos de las materias que enseñan y conocen el currículo nacional.

- Los docentes conocen los nuevos avances en su área.

- Los docentes comprenden las relaciones entre los contenidos que enseñan y los de otras disciplinas y pueden establecer relaciones entre estos contenidos y el mundo real.

- Los profesores saben cómo enseñar su materia y manejan diversas estrategias pedagógicas que son congruentes con las metas de aprendizaje.

Sin embargo, el conocimiento y las habilidades necesarios para enseñar cada materia no fueron bien especificados en el marco de buena docencia, por lo cual fue necesario ser más específicos acerca del conocimiento que los profesores principiantes deberían poder demostrar en las materias que se esperaba que enseñaran, es decir, en lenguaje, matemáticas, ciencias y estudios sociales, así como también lo que deberían saber acerca de cómo enseñar esas materias.

En el año 2011, el Gobierno chileno publicó los nuevos estándares nacionales para los profesores de primaria egresados, los que especificaban el conocimiento y las habilidades necesarios para enseñar cada tema a los alumnos de primaria. Cabe señalar que Chile es uno de los pocos países que ha desarrollado estándares con este nivel de esta profundidad. Los responsables de esta iniciativa fueron diferentes equipos de trabajo conformados por profesores, formadores de profesores y especialistas en contenido. También se desarrollaron pruebas nacionales alineadas con los estándares que los profesores deberían responder al final de su capacitación inicial como docentes. Los docentes que deseen postular a cargos en escuelas 
que reciben financiamiento público deberán aprobar necesariamente estas pruebas.

Así mismo, el Gobierno encargó el desarrollo de estándares para egresados de programas de formación docente secundaria en materias como historia, matemáticas, lenguaje, química, biología y física y actualmente se están desarrollando estándares para egresados de programas preescolares.

\subsection{Características de estándares bien elaborados}

La NBPTS en EE.UU. y Chile representan ejemplos de estándares bien redactados que intentan elucidar profundamente el tipo de conocimiento que los profesores deberían tener acerca del contenido que se espera que enseñen y cómo ayudar a los estudiantes a aprenderlo: estándares para profesores titulados, en el caso de la NBPTS, y estándares para profesores principiantes, en el caso de Chile. Estos estándares forman una base más válida y útil que los estándares genéricos y sobre la cual desarrollar evaluaciones del conocimiento y del desempeño de los profesores.

Por ejemplo, es común encontrar una afirmación como la siguiente en los conjuntos de estándares genéricos: «Los profesores titulados usan una variedad de estrategias de docencia». Este tipo de afirmación no sirve como guía para el contenido de los programas de formación docente ni para la creación de métodos para evaluar el desempeño del profesor, puesto que se centra en lo que hace la docencia más que en lo que aprenden los estudiantes, además, queda poco claro qué es lo que se toma en cuenta para cumplir con los estándares e ignora el hecho de que las estrategias adecuadas dependen profundamente de lo que se enseña y de a quién se le enseña.

Lo que necesita saber un profesor de primaria respecto de las estrategias para enseñar a leer es muy distinto de lo que necesita saber un profesor de matemáticas de escuela secundaria respecto de las estrategias para enseñar cálculo, por ello los estándares para la docencia deben identificar estas diferencias. En este sentido, una nueva investigación indica la importancia del conocimiento pedagógico específico de un profesor acerca de un tema particular 
en la promoción del aprendizaje de los estudiantes (por ejemplo Baumert, Kunter, Blum, Brunner, Voss, Jordan, Klusmann, Krauss, Neubrand y Tsai, 2010). Hill, Rowan y Ball (2005) y usa la expresión «conocimiento de matemáticas para la docencia» para describir este tipo de conocimiento en el caso de los profesores primarios.

Como se mencionó anteriormente, los estándares bien redactados se asientan en una clara comprensión de lo que se considera como aprendizaje de calidad para los estudiantes en áreas de contenido o niveles de escolaridad particulares. Veamos, por ejemplo, el extracto de un estándar en un conjunto de estándares para profesores de ciencias desarrollado por la Asociación Australiana de Profesores de Ciencias (2002):

Estándar: Profesores de ciencias titulados comprometen a los estudiantes en la indagación científica.

Elaboración: Los profesores de ciencias con altos logros guian a sus estudiantes en la indagación activa, para que ella los lleve a observar y medir fenómenos, formular hipótesis, registrar datos y llegar a conclusiones tentativas congruentes con los datos recopilados. Sus estudiantes reflexionan acerca del conocimiento que surge y consideran formas de refinar la investigación. Analizan y evalúan la evidencia que han recopilado con el fin de verificar la validez de sus conclusiones.

Su docencia refleja el interés y el desafío de la aventura científica y su rigor distintivo. Enseñan y ejemplifican prácticas que permiten a sus estudiantes alcanzar conocimiento y experiencias en forma critica, reconocer problemas, hacer preguntas e intentar soluciones. Involucran activamente a los estudiantes en una amplia variedad de investigaciones científicas (...)

Este estándar se explaya para describir un elemento importante de lo que considera aprendizaje de calidad, las aulas científicas e invita claramente al profesor a mostrar cómo compromete en forma activa a los estudiantes para hacer ciencia en sus escuelas. ¿Qué tipo de evaluación se requiere? Evidentemente, no aquella del tipo de logro nacional, sino que se necesitan métodos de evaluación que reflejen la complejidad de lo que el profesor intenta hacer, es decir, se requiere 
evidencia de lo que están haciendo y aprendiendo los estudiantes como resultado directo de lo que enseña el profesor.

Vale destacar algunas características de un estándar como este. La primera es que indica una porción grande, significativa e importante del trabajo de un profesor de ciencias, o sea, constituye un ejemplo de las complejas metas educacionales que se intenta lograr. Lo anterior implica que no se trata de una competencia a nivel micro ni de un rasgo de personalidad, sino que es algo válido de pedir a un profesor de ciencias para que demuestre que es un profesor de logros.

La segunda característica es que el estándar aparece libre de contexto, en el sentido de que describe una práctica que, según muchos, deberían seguir los profesores de ciencias sin importar dónde está la escuela. Por definición, un estándar profesional se aplica a todos los contextos en los que trabajan los profesores (que no es lo mismo que decir que el contexto no afecta la práctica docente). Así, el estándar invita a que, sin importar dónde esté una escuela, se pueda comprometer a los estudiantes en la indagación científica y se considera probablemente una responsabilidad vital de los profesores de ciencias.

El tercer rasgo distintivo es que el estándar no es prescriptivo respecto de las estrategias de docencia ni de cómo comprometer a los estudiantes para «hacer ciencias» y «pensar científicamente», lo cual significa que no estandariza la práctica ni fuerza a los profesores en ningún tipo de limitación, puesto que existen muchas formas de comprometer a los estudiantes en la indagación científica. De este modo, aunque el estándar identifica un elemento fundamental de la buena docencia científica, no prescribe cómo cumplir con él y, de esa manera, también da espacio a la diversidad y a la innovación. Los profesores son invitados a mostrar cómo cumplen con este estándar y evidenciar cómo es que comprometen a los estudiantes en la indagación científica.

La cuarta característica se refiere a la manera en la que el estándar apunta hacia algo que es medible u observable, con lo cual es posible imaginar los tipos de evidencia que reunirá un profesor de 
ciencias durante el tiempo para demostrar que cumple su cometido mostrando evidencias del trabajo de los estudiantes, segmentos de videos, o bien, recurriendo a otros mecanismos.

El conjunto de estas características se aplica a estándares en todas las áreas de la docencia, ya sea que ella ocurra en escuelas primarias o en las secundarias. En resumen, y usando la docencia de ciencias nuevamente como ejemplo, podemos decir que los estándares bien redactados:

- Se asientan en conceptos guía claros del aprendizaje de calidad y de lo que significa hacer (por ejemplo ciencias);

- son válidos, es decir, representan lo que saben y hacen los profesores que promueven las oportunidades de aprendizaje de calidad para los estudiantes;

- identifican características únicas de lo que saben y hacen los profesores;

- delinean las dimensiones principales de desarrollo que la profesión espera de un profesor (por ejemplo de ciencias), es decir, lo que deberían mejorar (los profesores de ciencias) con el tiempo, con oportunidades adecuadas para el desarrollo profesional; y

- se pueden evaluar, es decir, señalan características y acciones potencialmente observables.

Cuando los profesores analizan su práctica a la luz de un estándar bien redactado como el anterior, eso les brinda muchas oportunidades para el desarrollo profesional, ya que el estándar genera preguntas tales como: ¿Me comprometo realmente con mis alumnos en lo que significa hacer ciencia? ¿qué significa la indagación científica?, entre otras interrogantes que le darán la ocasión de revisar su práctica.

En este contexto es importante no polarizar ni separar los estándares para fines de desarrollo y los estándares para fines de evaluación, como hacen algunos. El desarrollo de un profesor depende en gran parte de las opiniones y de las evaluaciones constructivas de su desempeño y, desde esa óptica, los estándares serán útiles para la reflexión y el desarrollo profesional en la medida en que sean útiles 
para evaluar el desempeño: mientras más luces arroje la evaluación, sea una autoevaluación o la opinión de los colegas, más útil será para mejorar su desempeño. El mejor desempeño en docencia, así como muchas habilidades, se alimenta de opiniones exactas e informativas. Hemos comprendido mucho mejor que la evaluación de diagnóstico es vital para la docencia eficaz en la sala (por ejemplo Black y Wiliam 2004), y que el proceso de evaluación puede ser un vehículo para el aprendizaje de los estudiantes, lo cual no es menos cierto para enseñar y aprender a enseñar, o bien, para ser un mejor profesor.

\section{Evaluación de profesores basada en estándares}

Cuando los estándares se van a usar para fines de evaluación y toma de decisiones como por ejemplo con fines de selección o certificación, existen tres pasos esenciales para su creación:

a) Definir lo que se va a evaluar, por ejemplo, lo que necesitan saber y hacer los profesores principiantes (esto es lo que intenta hacer el marco de estándares y suelen llamarse estándares de contenido);

b) desarrollar métodos válidos y congruentes para recopilar evidencia acerca de lo que sabe y puede hacer un profesor en relación con los estándares (tareas de desempeño); y

c) fijar estándares, lo cual depende de la creación de procedimientos confiables para evaluar esa evidencia y decidir si un profesor cumplió o no con lo requerido (esto dependerá de la creación de estándares de desempeño, además de los estándares de contenido).

En otras palabras, si los estándares se usan para evaluar a profesores para fines de alto interés como el egreso, la selección o la certificación, existen tres pasos fundamentales en su desarrollo, como se muestra en la Figura 11.

Sykes y Plastrik (1993) definen un estándar como «una herramienta de alta precisión para hacer juicios y tomar decisiones en un contexto de significados y valores compartidos». Esto nos recuerda que un conjunto completo de estándares debe apuntar no solo hacia lo que se mide, sino también a cómo se reunirá la evidencia respecto 
62 ESTÁNDARES DE EGRESO Y CERTIFICACIÓN INICIAL DOCENTE: LA EXPERIENCIA INTERNACIONAL - L. Ingvarson

de la capacidad y el desempeño y cómo se harán los juicios acerca de si estos se cumplen o no.

Figura 11: Marco conceptual para el desarrollo de evaluaciones basadas en estándares

\begin{tabular}{|c|c|c|}
\hline $\begin{array}{l}\text { Estándares de contenido } \\
\text { ¿Qué se está midiendo? }\end{array}$ & $\begin{array}{l}\text { Tareas de desempeño } \\
\text { ¿Cómo se medirá? }\end{array}$ & $\begin{array}{c}\text { Estándares de desempeño } \\
\text { ¿Cómo juzgaremos la evidencia? }\end{array}$ \\
\hline
\end{tabular}

En este sentido, los estándares se consideran plenamente desarrollados cuando queda claro cómo se usarán para juzgar el desempeño (Hattie, 2008). Un conjunto completo de estándares no solo debe describir lo que saben y hacen los buenos profesores (es decir, lo que se evaluará), sino que debe detallar además cómo se recopilará la evidencia acerca de la capacidad y el desempeño y cómo se elaborarán los juicios respecto de si se ha cumplido con los estándares.

El decatlón olímpico ofrece una buena ilustración de los pasos involucrados en el desarrollo de un sistema basado en estándares para evaluar el desempeño. El concepto detrás del decatlón es el gran deportista versátil. Los orígenes del decatlón se remontan a principios del siglo XX cuando el rey Gustavo V de Suecia le dijo al estadounidense Jim Thorpe, «usted, señor, es el deportista más grande del mundo». Aparentemente, el episodio provocó la reflexión de las personas, quienes se preguntaron que es lo que hace que un deportista sea versátil. La reflexión dio cuenta de que el concepto necesitaba una definición más precisa: ¿Qué deberían poder hacer los deportistas versátiles? Después de mucho debate, decidieron que los elementos principales que identificaban a un gran deportista versátil eran fuerza, velocidad, histamina, resistencia y perseverancia. En otras palabras, definieron lo que debería ser evaluado, como si se trata de verdaderos estándares de contenido.

El paso siguiente fue llegar a acuerdo acerca de cómo juzgar al deportista versátil, esto es, cómo evaluar la fuerza, la velocidad, etc. En este caso, ¿qué se debería pedir a un deportista como evidencia de que es bueno en lo suyo? De algún modo, se tomó la decisión de someter a los deportistas a diez pruebas en dos días y entonces el concepto entró en operación: el primer día, las pruebas incluyeron 
la carrera de 100 metros, el salto largo, el lanzamiento de la bala, el salto alto y la carrera de los 400 metros, mientras que el segundo día, las pruebas contemplaron la carrera de los 110 metros vallas, el lanzamiento del disco, el salto con garrocha, la jabalina y la carrera de los 1.500 metros.

El conjunto de diez pruebas se consideró una muestra suficiente de evidencia para emitir un juicio acerca de la capacidad total de un deportista. Sin duda, la elección de las pruebas debió haber sido de algún modo arbitraria (ipor qué 100 metros en lugar de 200?), pero como grupo estas garantizaron la existencia de más de un tipo de evidencia para cada uno de los elementos (es decir, los estándares), es decir, cuando se recopila evidencia acerca de cada elemento en más de una forma, aumenta la confiabilidad de la evaluación (este será un punto fundamental para recordar al momento de evaluar el desempeño de un profesor).

Finalmente, fue necesario fijar los estándares de desempeño de un deportista versátil, lo que significaba llegar a un acuerdo acerca del nivel de desempeño en cada prueba que cumplía con el estándar. Así, se establecieron varios «niveles de referencia» para cada prueba del decatlón (por ejemplo, si se corren 100 metros en 10,395 segundos se ganan 1.000 puntos, si se logran en 10,827 segundos se ganan 900 puntos, si se alcanzan en 11,278 se ganan 800 puntos y así sucesivamente para cada prueba).

El nivel general de desempeño se determina mediante la ponderación y la combinación del desempeño en todas las pruebas, por lo que los estándares de desempeño no solo necesitan especificar lo bien que debe hacerlo el deportista en cada prueba para calificar, sino que deben especificar, además, lo bien que lo deben hacer en todas las pruebas en un promedio para clasificar como un buen deportista versátil. Los deportistas deben participar en el mismo conjunto de eventos, no hay otra opción; sin embargo, el buen desempeño en una prueba puede compensar un desempeño deficiente en otra.

Como esperamos haber mostrado, el decatlón representa un buen ejemplo de todo lo que está involucrado al momento de establecer un sistema de certificación congruente a nivel nacional. Es difícil imaginar 
que el decatlón hubiera tenido éxito si cada país hubiera podido decidir acerca de sus propias pruebas y estándares de desempeño, así como es igualmente difícil imaginar que pudiera surgir, en cada región del mundo, un buen sistema para la certificación de profesores titulados o egresados, o bien, que la autoridad de educación local desarrollara sus propios métodos de evaluación, usando los estándares nacionales solo como un marco: un sistema de certificación para cualquier profesión necesitará de métodos de evaluación congruentes y de la clasificación de esas tareas de evaluación.

Pasos similares al descrito para el caso del decatlón se aplican a cualquier sistema que pretenda ofrecer evaluaciones de docencia válidas, confiables y justas (Gitomer, 2009; Pearlman, 2008; Kennedy, 2010). Además, es necesario elaborar un marco genérico de estándares antes de poder usarlos para evaluar el conocimiento y la práctica del profesor, es decir, necesitan explicar lo que se espera que sepan los profesores en varias áreas en torno a lo que enseñan y a lo que aprenderán sus estudiantes.

Es importante observar que los estándares incluyen diferentes tipos de conocimiento, prácticas y responsabilidades profesionales y, en forma ideal, los métodos elegidos para la evaluación necesitan cubrirlos todos (se sabe que esto no será posible a nivel de egresados). Por lo tanto, con el fin de maximizar la confiabilidad de cualquier decisión basada en los estándares, será fundamental usar varios métodos de evaluación distintos y diferentes evaluadores.

Cuando sea posible, cada método debería entregar evidencia respecto de varios estándares a la vez. Del mismo modo, sería ideal que las tareas de evaluación fueran válidas o «auténticas», es decir, que se basen en porciones significativas del trabajo común que realizan los profesores al enseñar su disciplina. Por ejemplo, deberían representar lo que un profesor de ciencias necesita saber y hacer para promover las oportunidades de aprendizaje de calidad para que los estudiantes aprendan ciencias. Los mejores tipos de evidencias para este fin han sido los portafolios de ejercicios altamente estructurados y ejercicios centrados en la evaluación, ya que ellos entregan evidencia pertinente acerca de varios estándares a la vez. 


\section{Formación docente basada en estándares: avances internacionales}

Existe una tendencia internacional hacia el uso de estándares para guiar y acreditar programas de formación docente (DarlingHammond y Bransford, 2005; Wise, Ehrenberg y Leibbrand, 2008; Kennedy, 2010; Ingvarson, Elliott, Kleinhenz y McKenzie, 2006). Los países que han adoptado enfoques basados en estándares para la formación docente a algún nivel incluyen, entre otros, a Australia, Canadá, Chile, Inglaterra, Alemania, Jordania, Nueva Zelanda, Filipinas, Escocia, Singapur, Tailandia y EE.UU.

La mayoría de estos países estableció organismos a nivel nacional o estatal responsables de la creación y aplicación de estándares para la acreditación de programas de formación docente y la certificación de los nuevos profesores. El Consejo General de Docencia en Escocia es un ejemplo; el Instituto Victoriano de Docencia en Australia es otro. Un informe de Eurydice, Aseguramiento de la calidad en la formación docente de Europa (Eurydice, 2006) entrega un resumen integral de los procedimientos para evaluar y acreditar la formación docente inicial y en servicio en países europeos.

Como hemos dicho anteriormene, los estándares buscan definir el conocimiento, las capacidades y los valores que deberían obtener los futuros profesores gracias a sus programas de formación docente. Por ende, los estándares dan a los proveedores de formación de profesores una clara dirección acerca de las oportunidades para aprender que deberían ofrecer sus programas, sin prescribir la forma en la que deberían preparar a los profesores. Así mismo, los estándares muestran aquello que se espera que los estudiantes demuestren, es decir, lo que saben y pueden hacer antes de que sean idóneos para unirse a la profesión. Desde esta perspectiva, representan una base más sólida para evaluar y acreditar los programas de formación docente, a diferencia de los enfoques tradicionales centrados en contenidos y aportes de cursos. Ello, porque al enfocarse en los resultados, fomentan la diversidad y la innovación en la formación docente, ofreciendo una base para realizar investigación acerca de la eficacia 
relativa de los diversos enfoques para ayudar a los nuevos profesores a cumplir con los estándares.

Así, diremos que los componentes centrales en el sistema de formación docente basado en estándares incluyen:

a) Estándares que describen lo que deberían saber y hacer los profesores como resultado de su preparación y, por ende, ofrecer una guía para el aprendizaje profesional.

b) Un programa coherente para el aprendizaje profesional en donde individualmente cada uno de los cursos que lo componen se justifique en términos de cómo permite a los estudiantes cumplir con estándares particulares para la docencia y, en forma colectiva, los mismos cubran todos los estándares.

c) El egreso del programa y la certificación se basan en una variedad de tareas reales y evaluaciones de desempeño que, en conjunto, entregan evidencia confiable de que los estudiantes cumplen con todos los estándares.

d) La acreditación de programas de formación docente es realizada por un cuerpo profesional independiente y se basa en evidencia válida y confiable de que los egresados cumplen con los estándares para la certificación y el ingreso pleno a la profesión.

Juntos, estos componentes forman un sistema de elementos que se respaldan mutuamente y que fortalecen los programas de formación docente, pero solos, sus efectos sobre estos programas serán mínimos, es decir, si sacamos uno solo, la capacidad del sistema para apoyar la preparación eficaz de profesores disminuye.

En teoría, el énfasis que pone la formación docente basada en estándares sobre los resultados abre una mayor oportunidad para la innovación y la experimentación que los enfoques tradicionales orientados hacia la acreditación, los que tendían a centrarse más en la revisión de insumos tales como la naturaleza del contenido del curso, las listas de lectura, las tareas, etc. Uno de los principales objetivos de los estándares del Consorcio Interestatal de Apoyo y Evaluación de Profesores (InTASC), por ejemplo, es apoyar la reforma a la preparación y al desarrollo de profesores. 


\subsection{Fundamentos de la formación docente basada en estándares}

Quizás la principal justificación para el cambio hacia una formación docente basada en estándares sea mejorar el diseño de los programas de formación docente, haciéndolos más congruentes con lo que se sabe acerca de «la forma en la que los profesores aprenden y se desarrollan como profesionales» y cómo se pueden convertir en «expertos adaptables» (por ejemplo Hammerness, Darling-Hammond, Bransford, Berliner, Cochran-Smith, McDonald y Zeichner 2005: 358).

Aunque no existe una forma única y mejor de diseñar programas de formación docente, Darling-Hammond, Hammerness, Grossman y Shulman (2005) identifica varias consideraciones al respecto, la más importante de ellas es la congruencia del programa. En efecto, una característica de los programas congruentes es que las metas y los objetivos de cada curso dentro del programa se describen en términos de cómo contribuyen a ayudar a los estudiantes a cumplir estándares particulares. La congruencia dentro de los programas de formación docente es evidentemente importante y un marco de estándares compartido ayuda a promover esto.

En este mismo sentido es más probable que los cursos dentro de programas basados en estándares tengan una secuencia cuidadosa y se respalden mutuamente, ya que son guiados por una visión congruente y un modelo de buena docencia, la que se reseña en forma constante. La congruencia se refiere, además, a los sólidos vínculos entre el trabajo formal del curso, las experiencias de la escuela y las tareas. Por ejemplo, cuando los supervisores de la escuela comparten una comprensión común con el personal de la universidad en relación con los estándares que se usarán para ayudar a los profesores estudiantes y dan sus opiniones acerca de su desempeño: la experiencia en la escuela comienza primero y se produce en forma constante y los estudiantes deben demostrar cómo han aplicado lo que aprenden actualmente en el trabajo de curso en los escenarios escolares.

Darling-Hammond et. al. (2005) han revisado algunos estudios de investigación que muestran que los programas de formación docente guiados por estándares tienen un mayor impacto en los 
«conceptos y prácticas iniciales de los futuros profesores» que aquellos que siguen siendo una recopilación de cursos relativamente desconectados. Al respecto, un nuevo estudio internacional acerca de la formación docente en diecisiete países también mostró una relación importante entre la congruencia del curso y las percepciones de preparación entre los nuevos profesores (Ingvarson et. al., no publicado).

Una característica de los programas de formación docente basados en estándares es el esfuerzo que ponen sus diseñadores en asegurar que la preparación de los profesores se base en una asociación genuina entre los docentes en ejercicio y el personal de la universidad, una relación que se refleje tanto en la toma de decisiones, como en la distribución de financiamiento para la formación.

Los equipos encargados de elaborar los programas también hacen explícitos los principios fundamentales de la pedagogía sólida ante los cuerpos de acreditación (es decir, los estándares), así como también los métodos que usarán para garantizar que los futuros profesores aprenderán a implementarlos. Esto no se debería interpretar como una docencia estandarizada, puesto que un conjunto de estándares bien elaborado ofrece un marco pedagógico para reflexionar en torno a la labor de los profesores y constituye una herramienta flexible que permite que los egresados se sientan bien preparados para las exigencias de la docencia.

Otra consideración importante respecto del diseño nace de la investigación que demuestra la importancia central del conocimiento del contenido para la docencia eficaz (por ejemplo, Hill et. al., 2005; Goulding, Rowland y Barber, 2002; Kelcy, 2011; Kersting Givvin, Thomson, Santagata y Stigler, 2012). Esto incluye oportunidades para que los nuevos profesores adquieran una profunda comprensión de la materia que se espera que enseñen, acompañados por métodos de docencia y evaluación específicos al contenido, así como también el conocimiento acerca de las dificultades que suelen tener los estudiantes al aprender ese contenido y cómo abordarlas. En este sentido, en los últimos quince años ha habido un cambio notorio en Australia, desde cursos de pedagogía genérica hasta cursos centrados en el contenido, 
cambio que se puede atribuir en parte a la introducción de estándares para la docencia.

Por otra parte, hay evidencia de estudios que indican que la atención en el contenido y en la forma en la que los estudiantes lo aprenden es una característica clave de los programas de formación docente que tienen un mayor impacto en el aprendizaje de los estudiantes (por ejemplo Hawley y Valli, 1999; Kennedy, 1999). Los estándares basados en la investigación suelen llamar la atención sobre la importancia de garantizar que los programas proporcionen oportunidades suficientes para aprender el contenido en este sentido. Idealmente, los estándares se basan en síntesis de investigaciones sobre docencia y aprendizaje en áreas de contenido particular.

\subsection{Acreditación de Programas de Formación docente}

El informe de 2005 de la OCDE Teachers matter (OCDE, 2005) llamó la atención sobre las inquietudes generales concernientes a la oferta de profesores con talento en muchos países, en particular en áreas como matemáticas y ciencias. También puso el foco en la inquietud de muchos países por el hecho de que las disposiciones para evaluar y acreditar a las instituciones y programas de formación docente son débiles y tienen poco impacto en la calidad de este tipo de formación. Los autores argumentan al respecto que los criterios de acreditación deberían concentrarse en los resultados de los programas de formación docente y en estándares que describan lo que deberían saber y hacer los profesores principiantes como resultado de su capacitación.

En forma similar, el informe de la OCDE demuestra la creciente tendencia de los países de introducir políticas que exigen la certificación de los profesores nuevos, además de la culminación de programas de formación docente, antes de que puedan ingresar de lleno a la profesión. La certificación docente basada en estándares profesionales fue una palanca normativa que los gobiernos usaban cada vez más para ejercer influencia en los programas de formación docente y alinearlos en forma más estrecha con las necesidades de las escuelas. 
Una característica de los programas de formación docente basados en estándares es que las decisiones de egreso y de certificación inicial se basan cada vez con mayor frecuencia en la evidencia de que los estudiantes pueden cumplir con estándares de desempeño, en vez de las formas tradicionales de evaluación usadas en cursos y exámenes de la universidad (Wilson y Youngs, 2005). En este sentido, organismos tales como el Consejo Nacional para Acreditación de la Formación docente (NCATE) en EE.UU. han estado promoviendo durante muchos años la formación docente basada en estándares y un cambio hacia la acreditación basada en resultados (Wise et. al. 2008).

Así mismo, los últimos quince años han visto el desarrollo de métodos más sofisticados para evaluar el conocimiento del profesor respecto del contenido pedagógico y el desempeño según estándares para la docencia (por ejemplo Gitomer, 2009; Kennedy, 2010). El Consejo Nacional de Investigación en EE.UU. publicó un informe en 2001 acerca de las pruebas usadas para la aprobación de profesores en EE.UU. (National Research Council, 2001), las que mostraron que estándares bien redactados proporcionan un modelo o estructura que refleja la dinámica de lo que significa pensar y actuar como un profesor, lo que incluye el conocimiento de los estudiantes y sus necesidades, la selección de metas y actividades que valgan la pena para cumplir por dichas metas, la evaluación de lo que se ha aprendido y una reflexión en torno a la docencia.

Por otro lado, nuevas formas de evaluación como las desarrolladas por el proyecto Evaluación del Desempeño de Profesores de California (PACT) (Wei y Pecheone, 2010), o el proyecto Muestra de Trabajo del Profesor de la Western Oregon University (Shalock, Shalock y Girod, 1997) comprometen a los profesores-estudiantes en el diseño de unidades de trabajo, analizando su aprendizaje durante el tiempo y evaluando su propio desempeño según estándares. Los programas basados en estándares suelen poner a los futuros profesores en la función activa de crear un portafolio de evidencia que muestre cómo los cumplen y reflexionan acerca de esa evidencia. Esto es congruente con la investigación respecto de los modos eficaces de desarrollo profesional (Borko, 1997). De esta forma, los estándares para la docencia ofrecen un mapa o esquema cognitivo que ayuda a 
los futuros profesores a comprender las principales dimensiones de la buena docencia y cómo poder alcanzarla.

El Recuadro 2 muestra un ejemplo de cómo se incorporan las evaluaciones basadas en estándares en el Programa de Formación docente de la Universidad de Stanford en EE.UU. para ayudar a los estudiantes a cumplir con las expectativas de desempeño de la docencia establecidas por el Consejo de California para la Acreditación de Profesores. Otro avance interesante a favor de un enfoque orientado hacia los resultados en la formación docente es el trabajo de Deborah Ball y su equipo en la Universidad de Michigan. Su proyecto, llamado TeachingWorks, busca identificar un conjunto de habilidades principales que los profesores egresados deberían desarrollar, en tanto que los programas de formación docente se construirían sobre la base de estas «prácticas altamente motivadoras», ofreciendo materiales y recursos para apoyar el desarrollo de esas habilidades y métodos para evaluar su logro.

La acreditación externa de un organismo profesional independiente, en tanto, representa un componente clave de los sistemas de formación docente basados en estándares. Organismos como el Consejo Nacional para la Acreditación de la Formación docente (NCATE) en EE.UU. han promovido la formación docente basada en estándares, así como también un cambio hacia la acreditación basada en resultados (Wise et. al., 2008). Inglaterra, por ejemplo, tiene notoriamente uno de los sistemas de formación docente más regulados del mundo, pero a diferencia del NCATE, cuenta con una participación limitada por parte de la profesión o de los organismos profesionales en cuanto a la creación de sus estándares, o bien, de su funcionamiento. El gobierno en China (Taipéi) delega una responsabilidad considerable del sistema de acreditación en académicos y educadores de profesores, aunque se trata de uno de los pocos ejemplos de sistemas que tienen programas de educadores de profesores realmente desacreditados (Ingvarson et. al., no publicado).

Un informe de McKinsey y Company (Barber et. al., 2007) entregó conclusiones similares a las del estudio ETS anteriormente citado. Este informe llamado How the world's best performing school 
systems come out on top, también encontró una relación entre las políticas concernientes a la calidad docente y los resultados de los estudiantes, esta vez en relación con las mediciones PISA del logro académico.

Recuadro 2: Evaluación integrada en el programa de formación docente de Stanford (extracto del informe STEP para NCATE)

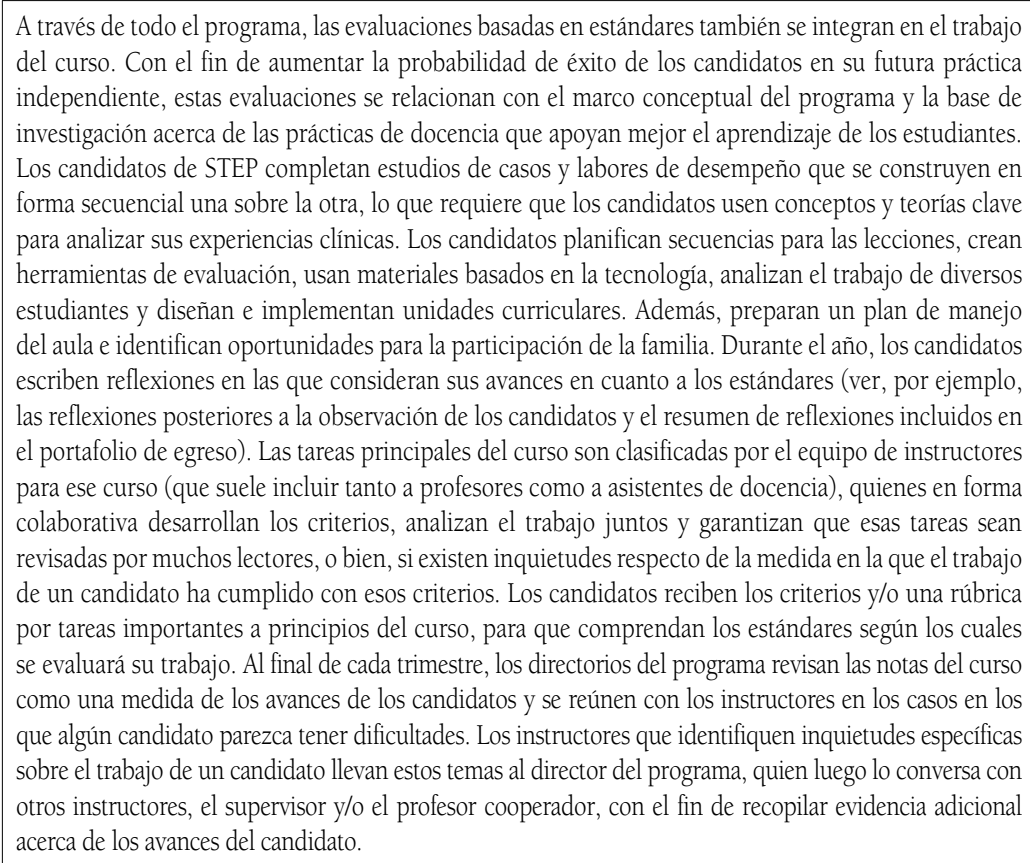

Varios estudios han mostrado las debilidades en la formación docente en EE.UU., lo que en parte podría explicar la tendencia hacia la utilización de enfoques basados en estándares. Al respecto, un estudio llevado a cabo por el Consejo Nacional sobre Calidad Docente (Greenberg y Walsh, 2008), No Common Denominator: the Preparation of Elementary Teachers in Mathematics by America's Education Schools encontró una amplia variación en las prácticas de formación docente en EE.UU., en especial en la proporción de cursos dentro de los programas que se concentran en enseñar matemáticas a los profesores de primaria y que deberán luego enseñar dicha materia. Por ejemplo, una reseña importante de la formación docente en EE.UU. indicó 
la falta de coherencia dentro de programas de formación docente y la necesidad de estándares «para contrarrestar el relativismo y la mentalidad todo vale que domina a la formación docente de hoy, lo que genera una multiplicidad de programas desunidos y conflictivos» (Levine, 2006: 106).

En 2005, el Panel sobre Investigación y Formación docente AERA publicó un informe llamado Studying teaching education, editado por Marilyn Cochran-Smith y Ken Zeichner. Un capítulo de Suzanne Wilson y Peter Youngs se concentra en la investigación relacionada con los procesos de rendición de cuentas en la formación docente, así como la certificación y la acreditación. Puesto que existen variaciones considerables de un estado a otro en cuanto a la aplicación coherente de estos procesos, Wilson y Youngs encontraron difícil llegar a conclusiones definitivas respecto de los efectos de la certificación y la acreditación, aunque sí pudieron concluir que la mayoría de los estudios que se centraban en las matemáticas mostraban una correlación positiva entre la certificación como profesor de matemáticas y el logro académico.

Wilson y Youngs (2005), por su parte, identifican una tendencia para desarrollar medidas basadas en estándares del conocimiento y desempeño del profesor principiante, entre las que se cuenta la serie de evaluaciones PRAXIS II y III (Dwyer, 1994) y las pruebas de Evaluación del Desempeño para Profesores de California (PACT), las que se desarrollan para un nuevo plan de aprobación en dicho estado (Wei et. al., 2010). Se espera que esta investigación ofrezca referencias más confiables para juzgar la calidad de los programas de formación docente y los egresados en el futuro.

\section{Referencias}

Barber, M. y M. Mourshed., (2007) How the best performing school systems come out on top. Reporte de McKinsey \& Co. Disponible en http:// mckinseyonsociety.com/downloads/reports/Education/Como_ hicieron_los_sistemas_educativos.pdf

Baumert, J.; Kunter, M.; Blum, W.; Brunner, M.; Voss, T.; Jordan, A.; Klusmann, U.; Krauss, S.; Neubrand, M. e Y. I. Tsai., (2010) «Teachers' mathematical knowledge, cognitive activation in the classroom, and 
student progress» en American Educational Research Journal. Volumen 47, número 1, marzo, pp. 133-180

Black, P. y D., William, (1998) Inside the black box: raising standards through classroom assessment. Londres, GL-Assessment. The Chiswick Centre.

Borko, H., (1997) «Teachers' developing ideas and practices about mathematics performance assessment: successes, stumbling blocks, implications for professional development» en Teaching and Teacher Education. Volumen 13, número 3, abril, pp. 259-278.

Brophy, J., (Ed.), (1991) Teacher's knowledge of subject matter as it relates to their teaching practice. Vol. 2. Londres, JAI Press Inc.

Chen, J., (2010) Teachers' conceptions of excellent teaching in Chinese middle schools. Tesis doctoral. Nueva Zelanda, Universidad de Auckland.

Cohen, D.K. y D, Ball, (1999) Instruction, Capacity, and Improvement. Reporte de investigación, serie RR-43. Filadelfia, University of Pennsylvania Graduate School of Education, Consortium for Policy Research in Education.

Council of Chief State School Officers., (2010) Model core teaching standards: a resource for state dialogue. Washington D.C., Council of Chief State School Officers.

Darling-Hammond, L. y J. Bransford., (Eds.), (2005) Preparing teachers for a changing world: what teachers should learn and be able to do. San Francisco, CA, Jossey-Bass.

Darling-Hammond, L.; Hammerness, K.; Grossman, P. y L. Shulman, (2005) «The design of teacher education programs» en Darling-Hammond, L. y J. Bransford. (Eds.), Preparing teachers for a changing world: what teachers should learn and be able to do. San Francisco, CA, Jossey-Bass, pp. 390-441.

Dwyer, C. A., (1994) Development of the knowledge base for the PRAXIS III: Classroom performance assessments assessment criteria. Princeton, Educational Testing Service.

EURYDICE, (2006) Quality assurance in teacher education in Europe. Bruselas, European Commission.

Gitomer, D., (Ed.) (2009) Measurement issues and assessment for teaching quality. Los Angeles, Sage.

Goulding, M.; Rowland, T. y B. Barber., (2002) «Does it matter? Primary teacher trainees' subject knowledge in mathematics» en British Educational Research Journal. Volumen 28. Número 5, octubre, pp. 689-704. 
Greenberg, J. y K. Walsh., (2008) No common denominator: the preparation of elementary teachers in mathematics by America's education schools. Reporte del National Council on Teacher Quality. Disponible en http://www.nctq. org/p/publications/docs/nctq_ttmath_fullreport_20090603062928. pdf

Hammerness, K.; Darling-Hammond, L.; Bransford, J.; Berliner, D.; CochranSmith, M.; McDonald, M. y K. Zeichner., (2005) «How teachers learn and develop» en Darling-Hammond, L. y J. Bransford (Eds.), Preparing teachers for a changing world: What teachers should learn and be able to do. San Francisco, California, Jossey-Bass, pp. 358-389.

Hanushek, E. A., (2004) Some simple analytics of school quality. Documento de trabajo número 10229. Cambridge, Massachusetts: National Bureau of Economic Research.

Hattie, J., (2008) Visible learning: a synthesis of over 800 meta-analyses relating to achievement. Londres, Routledge.

Hawley, W. y L. Valli., (1999) «The essentials of effective professional development: a new consensus» en Darling-Hammond, L. y G. Sykes (Eds.), Teaching as the learning profession. Handbook of policy and practice. San Francisco, California, Jossey-Bass Publishers, pp. 127-150.

Hill, H.; Rowan, B. y D. Ball, (2005) «Effects of teachers' mathematical knowledge for teaching on student achievement» en American Educational Research Journal. Volumen 42, número 2, verano, pp. 371-406.

Ingvarson, L.C.; Schwille, J.; Rowley, G.; Tatto, M.T.; Peck, R. y S.L. Senk., (No publicado) National policies and regulatory arrangements for the preparation of teachers in TEDS-M countries. Amsterdam, International Association for the Evaluation of Educational Achievement.

Ingvarson, L.C., y E. Kleinhenz., (2006) Advanced teaching standards and certification: A review of national and international developments. Reporte del Teaching Australia (Australian Institute for Teaching and School Leadership). Disponible en: http://www.teachingaustralia.edu.au/ta/ go/home/projects/standards.

Ingvarson, L., Elliott, A., Kleinhenz, E. y P. McKenzie. (2006) «Accreditation of teacher education: a review of national and international trends and practices in other professions. Report prepared for teaching Australia» (Australian Institute for Teaching and School Leadership Ltd) en Ingvarson, L.C. y J. Hattie. (Eds.) (2008) Assessing teachers for professional certification: the first decade of the national board for professional teaching standards. Amsterdam, the Netherlands: Elsevier Press. 
Irving, E., (2005) The development and validation of a student evaluation instrument to identify highly accomplished mathematics teachers. Tesis doctoral. Nueva Zelanda, Universidad of Auckland.

Kennedy, M., (Ed.) (2010) Teacher assessment and the quest of teacher quality. San Francisco, California, Jossey-Bass.

Levine, A., (2006) Educating school teachers. The Education School Project. Disponible en http://www.edschools.org/pdf/Educating_Teachers_ Report.pdf.

Meckes, L., (2011) Teaching standards in Chile. Santiago, Centro de Estudios de Políticas y Prácticas en educación (CEPPE), Universidad Católica, Chile.

National Research Council., (2001) Testing teacher candidates. Washington, DC, National Academy Press.

Organization for Economic Cooperation and Development. (2005) Teachers matter: attracting, developing and retaining effective teachers. Paris, Organization for Economic Cooperation and Development.

Organisation for Economic Cooperation and Development, (2010) Strong performers and successful reformers in education: lessons from PISA for the United States. Disponible en http://dx.doi. org/10.1787/9789264096660-en

Organisation for Economic Cooperation and Development., (2011) Building a high-quality teaching profession: lessons from around the world background report for the International Summit on the Teaching Profession. Paris: Organization for Economic Cooperation and Development.

Pearlman, M., (2008) «The evolution of the NBPTS scoring system» en Ingvarson, L.C. y J. Hattie, (Eds.), Assessing teachers for professional certification: the first decade of the National Board for Professional Teaching Standards. Amsterdam, Elsevier Press.

Reynolds, A., (1992) «What is competent beginning teaching? A review of the literature» en Review of Educational Research. Volumen 62, número 1, primavera, pp. 1-35.

Ryle, G., (1946) «Knowing how and knowing that» en Proceedings of the Aristotelian Society. Número 46. pp. 1-16

Shalock, H.D.; Shalock, M. y G. Girod., (1997) «Teacher work sample methodology as used at Western Oregon State College» en J. Millman, (Ed.), Grading teachers, grading schools: is student achievement a valid evaluation measure? Thousand Oaks, California, Corwin Press. 
Shulman, L.S., (1987) «Knowledge and teaching: foundations of the new reform» en Harvard Education Review. Número 57, febrero, pp. 1-22.

Sykes, G. y P. Plastrik., (1993) Standard setting as educational reform. Washington DC: ERIC Clearinghouse on Teacher Education.

Tatto, M. T.; Schwille, J.; Senk, S. L.; Ingvarson, L.; Rowley, G.; Peck, R.; Bankov, K.; Rodríguez, M. y M. Reckase, (2012) Policy, practice, and readiness to teach primary and secondary mathematics in 17 countries: findings from the IEA Teacher Education and Development Study in Mathematics (TEDS-M). Amsterdam, International Association for the Evaluation of Educational Achievement.

Wei, R.C. y R. Pecheone., (2010) «Assessment for learning in preservice teacher education: performance-based assessments» en Kennedy, M., (Ed.), Teacher assessment and the quest of teacher quality. San Francisco, California, Jossey-Bass

Wilson S. y P. Youngs., (2005) «Research on accountability processes in teacher education» en Cochran-Smith, M. y K. Zeichner (Ed.), (2005) Studying teacher education: the report of the AERA Panel on Research and Teacher Education. Washington DC, American Educational Research Association, pp. 591-644.

Wise, A.E.; Ehrenberg, P. y J. Leibbrand., (2008) It's all about student learning: assessing teacher candidates ability to impact P-12 students. Washington: National Council for Accreditation of Teacher Education.

Recibido: 30/10/2012

Aceptado:30/10/2012 